\title{
Chloride, Calcium Carbonate and Total Soluble Salts Contents Distribution for An-Najaf and Al-Kufa Cities' Soil by Using GIS
}

\author{
Sohaib Kareem Al-Mamoori - Laheab A. Jasem Al-Maliki - Khaled El-Tawel • \\ Hussain M. Hussain • Nadhir Al-Ansari (D) Mohammed Jawad Al Ali
}

Received: 12 August 2018/Accepted: 12 November 2018/Published online: 29 November 2018

(C) The Author(s) 2018

\begin{abstract}
The main objective of the paper is to create geotechnical maps for three soil chemical properties in An-Najaf and Kufa cities' soil by utilizing of GIS tools. This properties are the chloride concentration, calcium carbonate $\left(\mathrm{CaCO}_{3}\right)$ and total soluble salts where they affect the durability of reinforced structural elements. This paper provides an easy accurate way to represent soil properties levels for different depths of soil and create reliable database that will help engineers and decision makers. The data included
\end{abstract}

S. K. Al-Mamoori · M. J. Al Ali

Department of Environmental Planning, Faculty of

Physical Planning, University of Kufa, Najaf, Iraq

e-mail: sohaib.almamoori@uokufa.edu.iq

M. J. Al Ali

e-mail: mjkplaner@gmail.com

L. A. J. Al-Maliki

Department of Hydraulic Engineering Structures, Faculty of Water Resources Engineering, Green University of Al Qasim, Baghdad, Iraq

e-mail: laheab.almaliki@wrec.uoqasim.edu.iq

K. El-Tawel

Faculty of Engineering, Lebanese University, Beirut,

Lebanon

e-mail: khaled_tawil@ul.edu.lb

H. M. Hussain

Remote Sensing Center, University of Kufa, Najaf, Iraq

e-mail: hussainm.alshimmary@uokufa.edu.iq in this paper were collected for (464) boreholes with depths up to $35 \mathrm{~m}$ distributed on residential areas in all of An-Najaf and Kufa cities. Arc-Map of GIS 10.2.1 was used to produce the maps. It has been concluded that chloride content in the soil of the study area range from -0.01 to $0.99 \%$ and with an average of 0.5 . The maximum value found in at depth 4-6 $\mathrm{m}$ while the minimum value found in location at depth $4-6,8-10$ and 14-16 m. The chloride content in most of AnNajaf province has exceeded the permissible limit and

\section{H. M. Hussain}

Department of Geology, Faculty of Science, University of Kufa, Najaf, Iraq

N. Al-Ansari ( $\square)$

Department of Civil, Environmental and Natural

Resources Engineering, Lulea University of Technology,

Luleå, Sweden

e-mail: nadhir.alansari@ltu.se 
for all, this required taking percussions to protect foundations. While calcium carbonate content in the soil of the study area range from 53 to 0.18 and with an average of 26.6. The maximum and the minimum values found at depth $4-6 \mathrm{~m}$. Calcium carbonate content is within the permissible limits in most locations except in some locations.

Keywords Chloride content · GIS · Calcium carbonate $\left(\mathrm{CaCO}_{3}\right) \cdot$ Total soluble salts (TSS) · AnNajaf and Kufa cities - Geotechnical maps .

Reinforced concrete

\section{Introduction}

For any project to be done a complete soil investigations must be carried out, these investigations are very hard, expensive and time-consuming work. That's why, a need appear to reduce them as much as possible. The corrosion of steel reinforcement is one of the most important aspects in the durability of reinforced structural elements (Oh and Jang 2005) in addition to the failure of the concrete itself.

Chloride attack is the main cause of structural damage in reinforced structural elements which is triggered by the propagation of the chloride ions through the pore solution to the reinforcement level (Mallick et al. 2018). The chloride ions may be redounded by the ingredients of concrete mixture, such as aggregates, mixing water, cement, and Additives for concrete mixtures. Alternatively, they may spread into the sclerotic concrete from the surrounding environment (Karthick et al. 2016). The diffusion of chloride ions from the external environment through concrete to the reinforcement steel's surface, leads to the erosion of the precautionary layer and finally to reinforcement corrosion (Hirao et al. 2005). One such case is the spread of chloride ions from the surrounding soil into the hardened concrete in foundations or substructures (Maslehuddin et al. 2007). There are no guidelines or threshold limits for the chloride concentration in the soil and its influence on reinforcement corrosion (Maslehuddin et al. 2007). That is because chloride effect is dependent upon many factors such as type of cement used in the concrete mixed. Chloride does not affect the concrete or the reinforcement in direct way but it does make deterioration of concrete from cyclic freezing even worse if the concrete was so exposed (Niville 1995). The existence of chloride ions in concrete has the potential to boost erosion of reinforcement (Al-Amoudi et al. 2015). The major concern when dealing with reinforced structural elements exposed to soils containing chlorides is erosion of reinforcement bars. Concrete permeability will have a "time" effect, where if chlorides are founded in any but the smallest amounts and there is even a little moisture in the soil, then erosion of reinforcement steel will eventually occur (Ali 2017). With comparatively impermeable concrete, the start of erosion will only be belated, and that is all. When the ground water rose above the concrete foundations they will be affected by chemical effects associated with consecutive cycles of drying and moistening. At high water table the concrete saturate with water, and when it drops, part of the water evaporates from concrete causing salts crystallizing. A following water rise causes the concrete saturation again with water that contain higher salts concentration. The repetition of this process by time causes salts crystallization inside the concrete pores, if it is permeable, resulting in a swelling leading to develop cracks in concrete. There is an allowable size limit for these cracks for various types of exposure; these limits range from $0.1 \mathrm{~mm}$ for very extreme environment to $0.4 \mathrm{~mm}$ for moderate environment (Mallick et al. 2018).

Carbonation is another chemical process which leads to concrete degradation. This process is the interaction of calcium hydroxide $\mathrm{Ca}(\mathrm{OH})_{2}$, with carbon dioxide $\mathrm{CO}_{2}$ existing in the air. At first, carbonic acid is engender by carbon dioxide dissolving in the pore water, and it in turn reacts with calcium hydroxide to form calcium carbonate $\left(\mathrm{CaCO}_{3}\right)$ (Bonic et al. 2015). The greater permeability and porosity of concrete, the easier it is gets corroded (Glasser et al. 2008). However, the main result for concrete carbonation is the reduction of alkaline nature of concrete, which is a very important precondition for reinforcement corrosion (Bonic et al. 2015). Total soluble salts TSS is defined as all soluble soil salts, where the solubility of these salts varies by its nature. The degree of solubility is affected by the temperature, the $\mathrm{pH}$ value and the quantity of the dissolved carbon dioxide $\mathrm{CO}_{2}$, as well as evaporation and moisture (Atyah and Muir 2013). The importance of this test is to estimate the proportions of salts that cause the erosion of concrete and pipes and reinforcing steel. When their 
solubility is free in water, it has a significant impact on the degree of damage to the concrete (Emmanuel et al. 2012).

This study included collect, sort and analyze the data of (464) boreholes in the study area and for depths from 0 to $35 \mathrm{~m}$. The data were obtained from the National Center for Construction Laboratories \& Researches (NCCLR 2016)/Babylon laboratory reports (NCCLR).

\section{Methodology}

The methodology of this work include the steps listed in the flow chart shown in Fig. 1.

\subsection{The Study Area}

An-Najaf governorate is located at the southwest of Iraq away from the capital Baghdad of about $161 \mathrm{~km}$

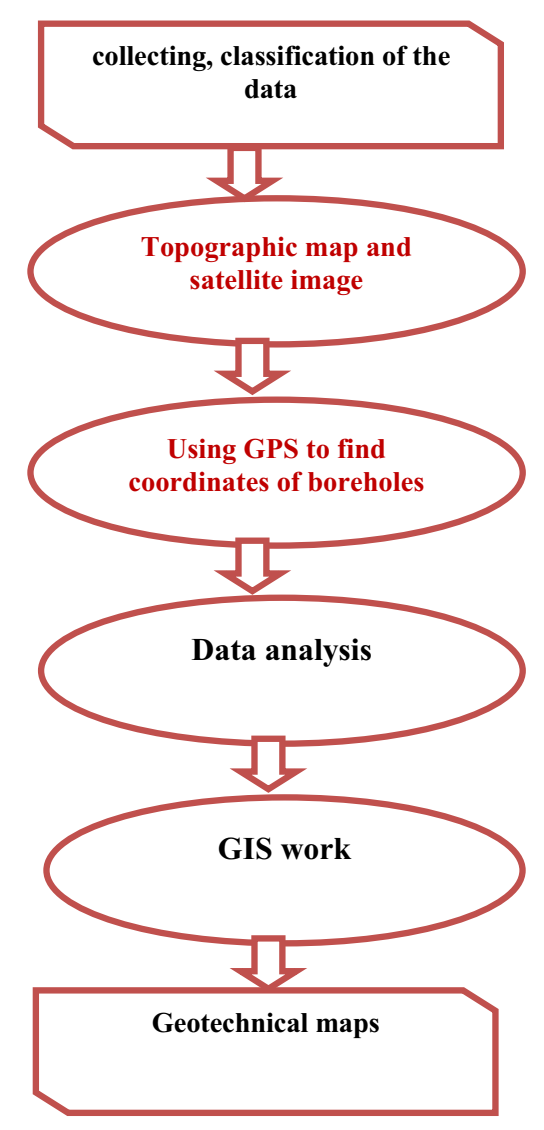

Fig. 1 The work flow chart

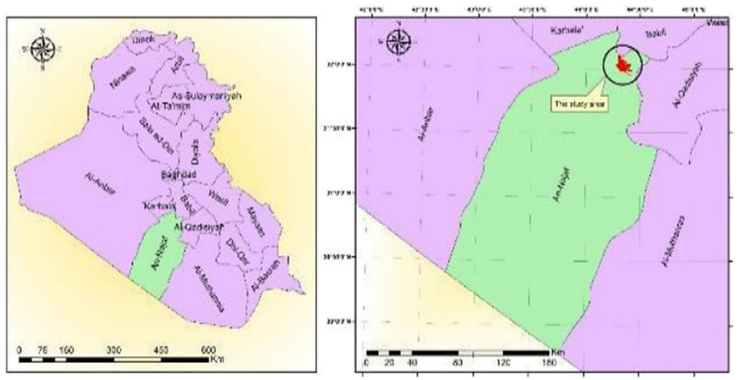

Fig. 2 Location of An-Najaf province

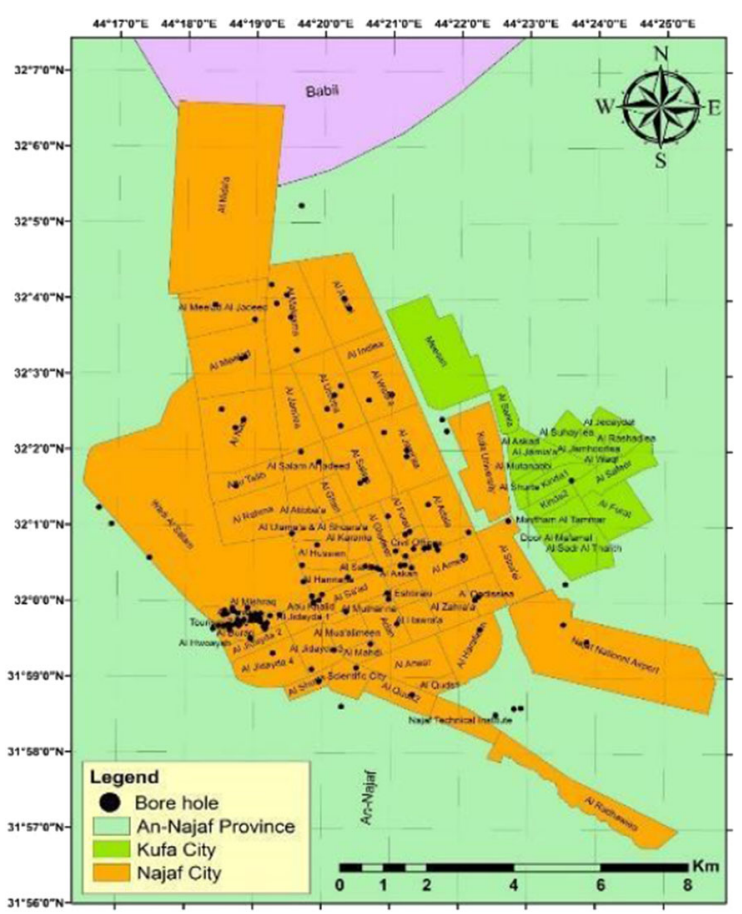

Fig. 3 Location of study area

southwest (Fig. 1). It has an area of about $29,000 \mathrm{~km}^{2}$ and comprise about $7.1 \%$ of the country total area (Mallick et al. 2018). It is located at the intersection of longitude $44^{\circ} 21^{\prime} 0^{\prime \prime}$ eastern and latitude $32^{\circ} 2^{\prime} 0^{\prime \prime}$ northern. This study involve parts of An-Najaf and Kufa cities that located between the coordinates $44^{\circ} 17^{\prime} 00^{\prime \prime}$ and $44^{\circ} 25^{\prime} 0^{\prime \prime}$ Eastwards and $32^{\circ} 7^{\prime} 0^{\prime \prime}$ and $31^{\circ}$ [the researcher]. Figure 1 shows the location of An-Najaf province. Figure 2 show the of study area and locations of boreholes (Fig. 3). 
Table 1 Neighborhoods numbering

\begin{tabular}{|c|c|c|c|}
\hline Num. & Neighborhood & Num. & Neighborhood \\
\hline 1 & Wadi Al Salam & 42 & Al Muthanna \\
\hline 2 & Al Askari & 43 & Abu Khalid \\
\hline 3 & Al Makrama & 44 & Al Mahdi \\
\hline 4 & Al Nasr & 45 & Al Meelad Al Jadeed \\
\hline 5 & Al Wafa'a & 46 & Al Radhawiea \\
\hline 6 & Abu Talib & 47 & Al Rahma \\
\hline 7 & Al Meelad & 48 & Najaf National Airport \\
\hline 8 & Al Jam'iea & 49 & Scientific City \\
\hline 9 & Al Urooba & 50 & Al Nida'a \\
\hline 10 & Al Indiea & 51 & Najaf Technical Institute \\
\hline 11 & Al Ghari & 52 & Kufa University \\
\hline 12 & Al Jami’aa & 53 & Meesan \\
\hline 13 & Al Salam & 54 & Kinda2 \\
\hline 14 & Al Salam Al jadeed & 55 & Maytham Al Tammar \\
\hline 15 & Al Atibba'a & 56 & Kinda1 \\
\hline 16 & Al Ansar & 57 & Al Shurta \\
\hline 17 & Al Harafieen & 58 & Al Mua'alimeen \\
\hline 18 & Al Quds2 & 59 & Al Askari \\
\hline 19 & Al Quds1 & 60 & Al Mutanabbi \\
\hline 20 & Al Sina'ei & 61 & Al Jamia’a \\
\hline 21 & Al Ameer & 62 & Al Jedaydat \\
\hline 22 & Al Adala & 63 & Al Waqf \\
\hline 23 & Al Hussien & 64 & Al Rashadiea \\
\hline 24 & Al Furat & 65 & Al Jamhooriea \\
\hline 25 & Al Ghadeer & 66 & Al Safeer \\
\hline 26 & Civil Offices & 67 & Al Furat \\
\hline 27 & Al Karama & 68 & Door Al Ma'amal \\
\hline 28 & Al Hannana & 69 & Al Sadr Al Thalith \\
\hline 29 & Al Sahha & 70 & Al Suhayliea \\
\hline 30 & Al Ulama'a \& Al Shuara’a & 71 & Al Sahla \\
\hline 31 & Al Zahra'a & 72 & Al Jidayda 1 \\
\hline 32 & Al Qadissiea & 73 & Al Jidayda 3 \\
\hline $6+33$ & Al Askan & 74 & Tourism Zone \\
\hline 34 & Al Sa'ad & 75 & Al Jidayda 2 \\
\hline 35 & Adan & 76 & Commercial Zone \\
\hline 36 & Al Mua'alimeen & 77 & Imam Ali Shrine \\
\hline 37 & Al Shurta & 78 & Al Buraq \\
\hline 38 & Al Jidayda 4 & 79 & Al Mishraq \\
\hline 39 & Al Hwoaysh & 80 & Al Emara \\
\hline 40 & Al Hawra'a & 81 & Al Sooq Al Kabeer \\
\hline 41 & Al Eshtiraki & & \\
\hline
\end{tabular}




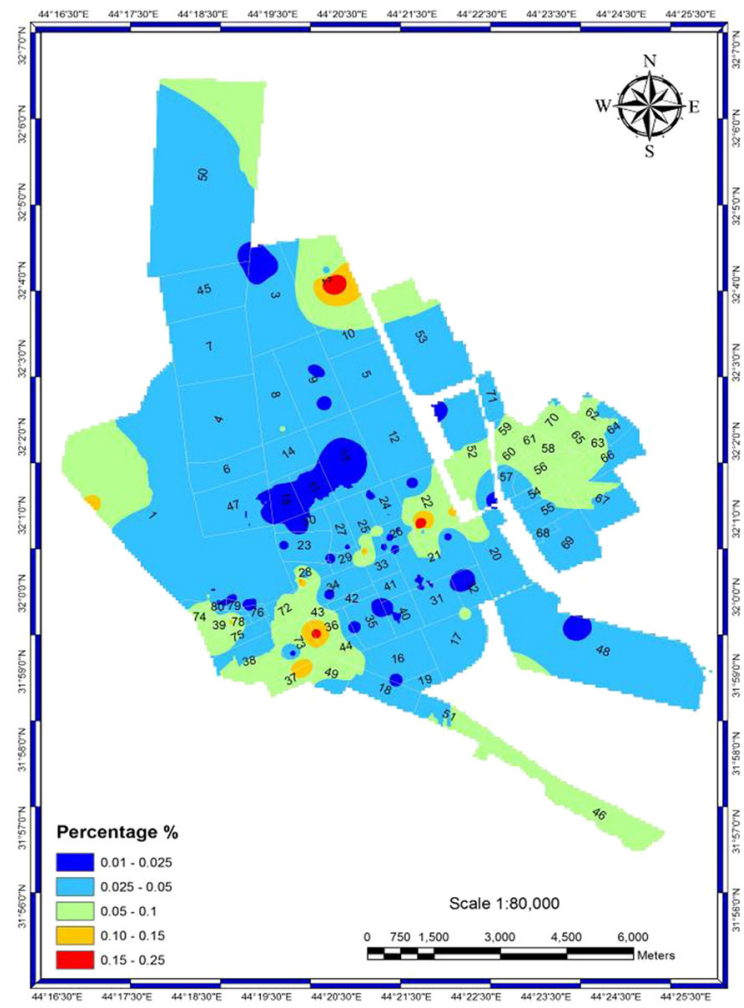

Fig. 4 Chloride concentration at depths 0-2 m

\subsubsection{An-Najaf City Soil Description}

Generally, An-Najaf City Soil consist of sand about $50-85.1 \%$ of the soil components, with some layers of clay and silty clay soil at various depths. SPT test values that dominated on most of the region are high and exceeds 50 blows. the most existing soil conditions are dense to very dense sand, and cemented sand. Angle of Internal friction $\varnothing$ range between $26.3^{\circ}$ and $41.2^{\circ}$ in most of the region (Ali and Fakhraldin 2016). The bearing capacity form SPT values ranged between 5 and $20 \mathrm{Ton} / \mathrm{m}^{2}$ in the studied region (Al-Maliki et al. 2018). Sulfate content in soil varies between 0.36 and $14 \%$ while in groundwater was between 84 and $239 \%$ (Al-Mamoori et al. 2017). Gypsum content is very high, it rang between 10 and 25\% (Al-Mamoori 2017).

\subsubsection{An-Najaf City Climate Description}

The study area is characterized by desert climate, that is a hot dry summer and cold winter (Iraqi Ministry of Planning, Central Statistical Organization). Summer temperatures in the study area range between 41 in

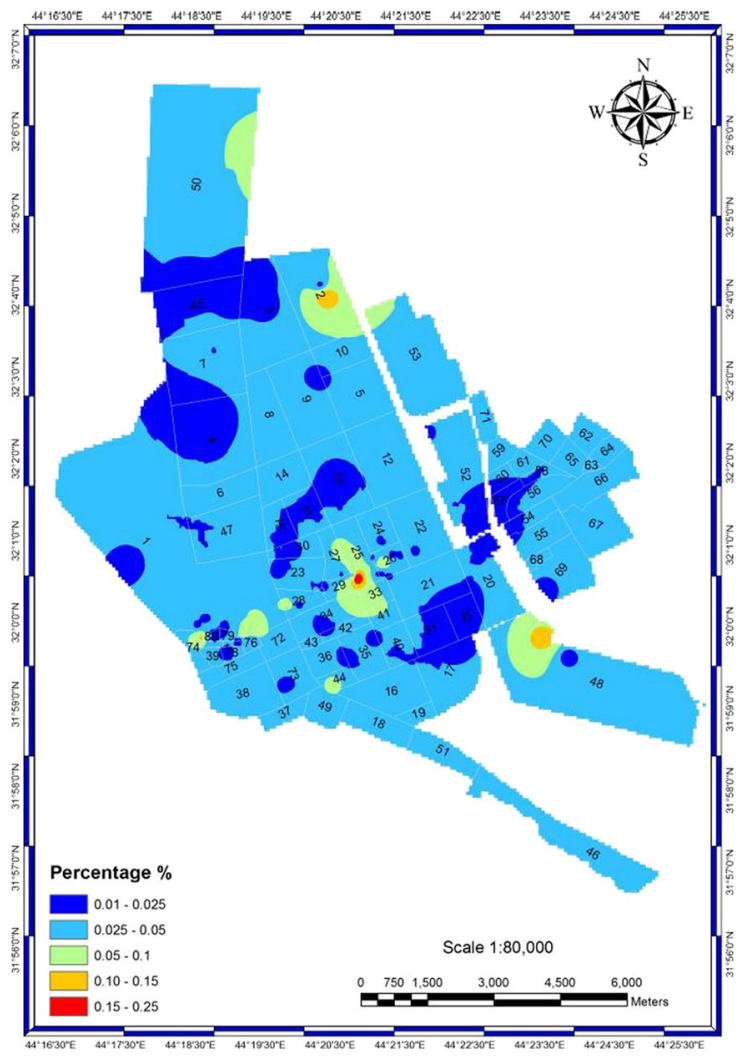

Fig. 5 Chloride concentration at depths 2-4 m

June, 44 in July and 43.4 in August, while the temperatures diminish in winter to 5 in December, 4 in January, and 5.6 in February. Rainfall is limited from October to May and unevenly distributed. The total annual rainfall is 101, 112, $121 \mathrm{ml} / \mathrm{cm}^{2}$ (Environmental Statistics Report, Iraqi Ministry of Planning). The climate is an important factor affecting the quality of soil, water and air. The increase in the amount of rain precipitation leads to the filtration of water through the soil layers, washing salts and then the rise of groundwater levels, in addition to the reduction of some elements in soil and groundwater. On the contrary the lack of rainfall and increase the temperature, especially in the summer, lead to water evaporation, soil dryness, lower ground water levels and increased crystallization of salts (Mahmoud and Jaafar 2017).

\subsection{Research Method}

In order to achieve the objective of the study, the following steps were taken 


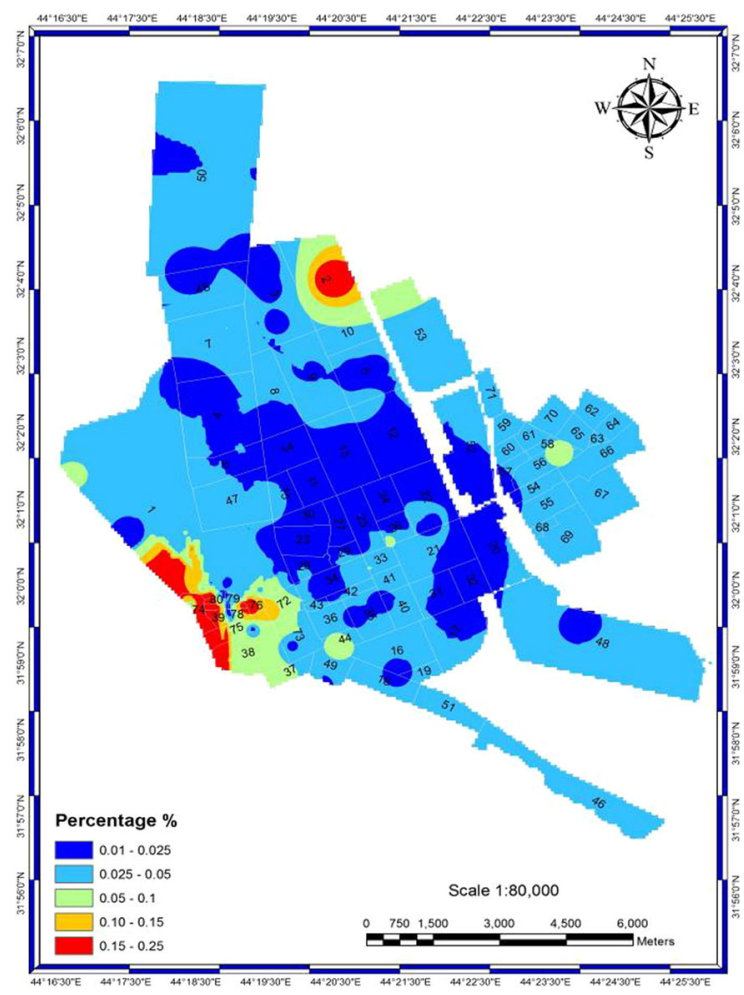

Fig. 6 Chloride concentration at depths 4-6 m

1. The used data were obtained from the National Center for Construction Laboratories \& Researches (NCCLR)/Babylon laboratory reports. Babylon laboratory is a department that attached to the National Center for Construction Laboratories \& Research (NCCLR), that is part of the Ministry of Construction and Housing authority. The laboratory is effective since its emergence in 1977. Therefore; information about the province of An-Najaf and all areas are available in this center.

2. After collecting the data, the exact locations for each borehole were obtained using GPS (see "Appendix").

3. The data obtained was arranged in tabular format within the Excel program including the borehole location and concentrations for each depth.

4. Position the boreholes sites on the latest administrative map of the study area.

5. In order to e facilitate and simplify the produced maps each Neighborhood was replaced by a certain number. Neighborhood's names and numbers are listed in Table 1.

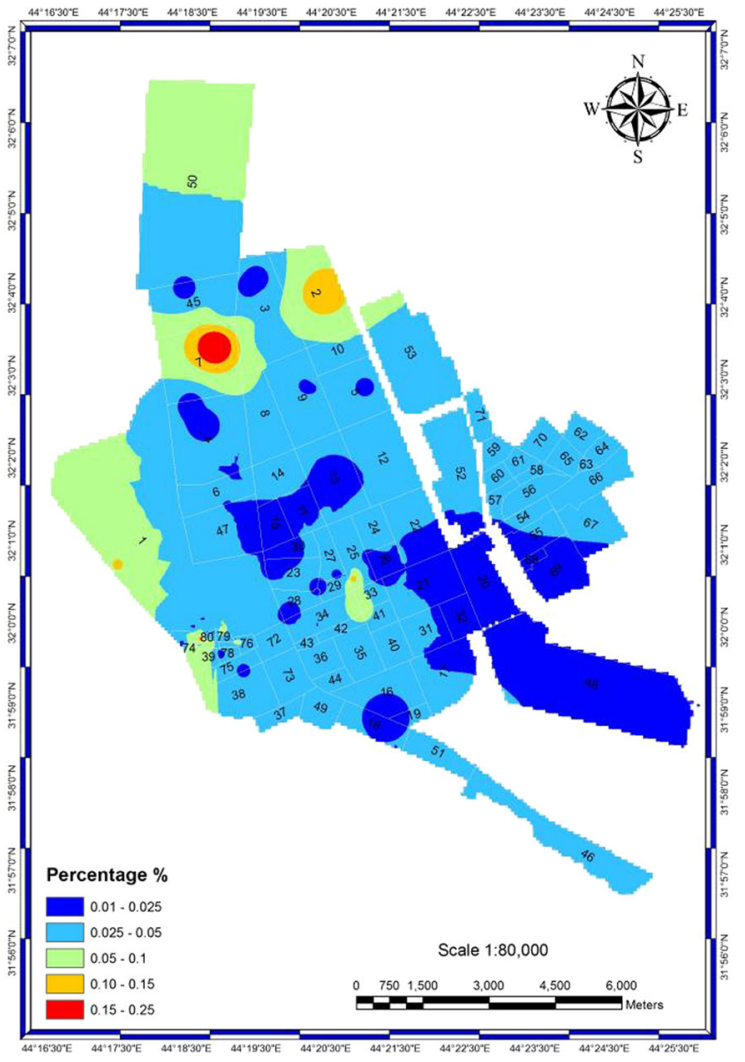

Fig. 7 Chloride concentration at depths 6-8 m

This research data were obtained from 464 boreholes spread in An-Najaf and Al-Kufa cities and for depths from 0 to $35 \mathrm{~m}$.

\subsection{Software Program}

The Geographic Information System (GIS) is an integrated geographic information system published by the Environmental Systems Research Institute (ESRI). Arc Map is the central part of the program that has many tasks, such as mapping, editing, displaying, present digital data, working with layers, add elements to the maps such as scale and map legend. This research included sorting and categorizing the collected date in Excel program, the sorted data include the names of the sites and the sites' features, as well as tables containing the data that we will use in the analysis process. Then the Excel file imported to the GIS program to analyze and produce maps using Inverse Distance Weighted (IDW) tool for this aspect. IDW is an interpolation method that use points with 


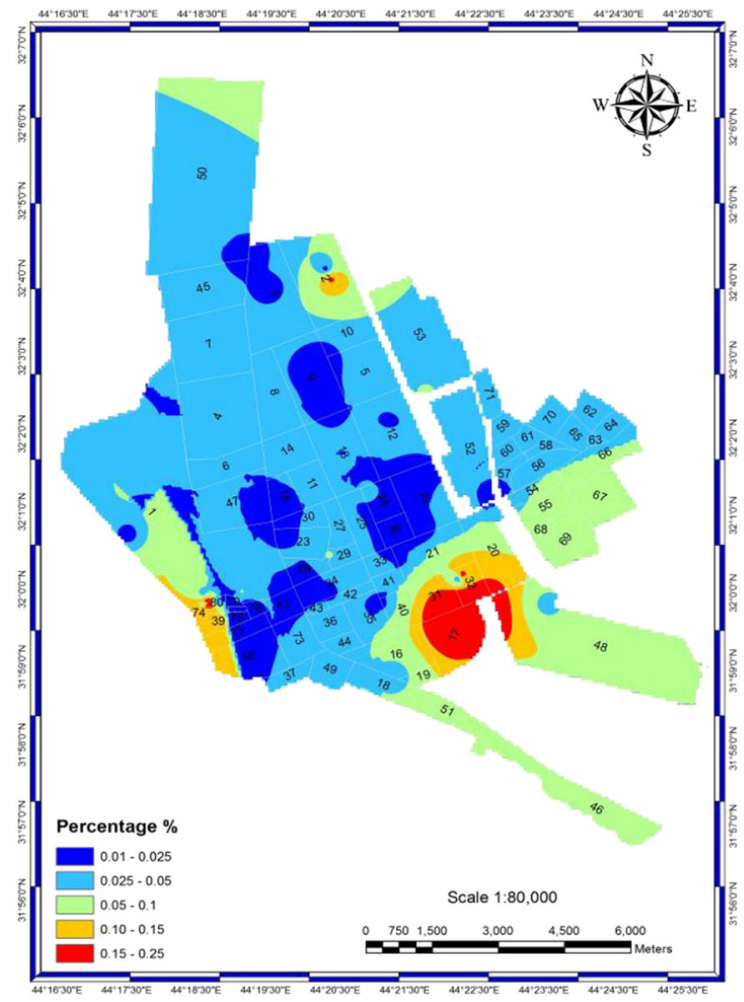

Fig. 8 Chloride concentration at depths $8-10 \mathrm{~m}$

known values or sample points to estimate values at other unknown points. In GIS program there are eight interpolation methods. Inverse Distance Weighting IDW is one of these methods which assumes that each input point has a local influence that diminishes with distance. The reason of using this method is because it can estimate extreme changes in terrain such as: Cliffs, Fault Lines, dense evenly space points are well interpolated (flat areas with cliffs) and can increase or decrease amount of sample points to influence cell

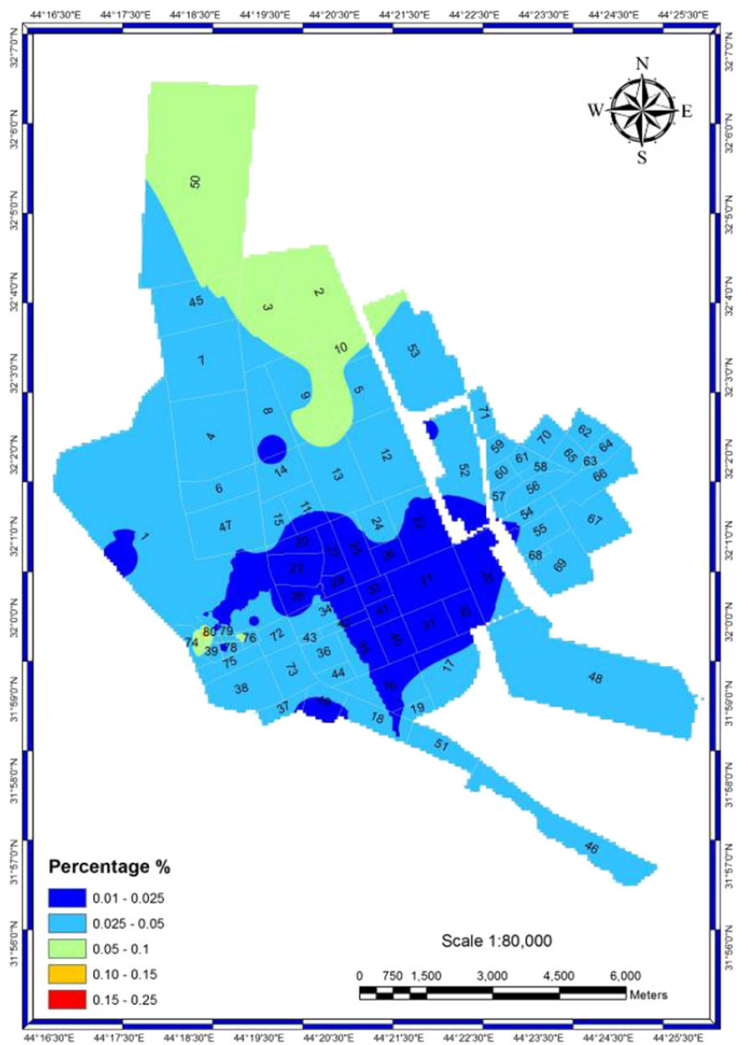

Fig. 9 Chloride concentration at depths 10-12 m

values. Finally geotechnical maps were prepared for each soil property. In this paper the version GIS (10.2.1) of the program was used.

The Geographic Information System (GIS) have tools that can help make, manage, analyze, and visualize any data specially these related to civil and urban infrastructures. It helps engineers and decision maker to transform the geotechnical data into practicable information that are useful for the reliable and 


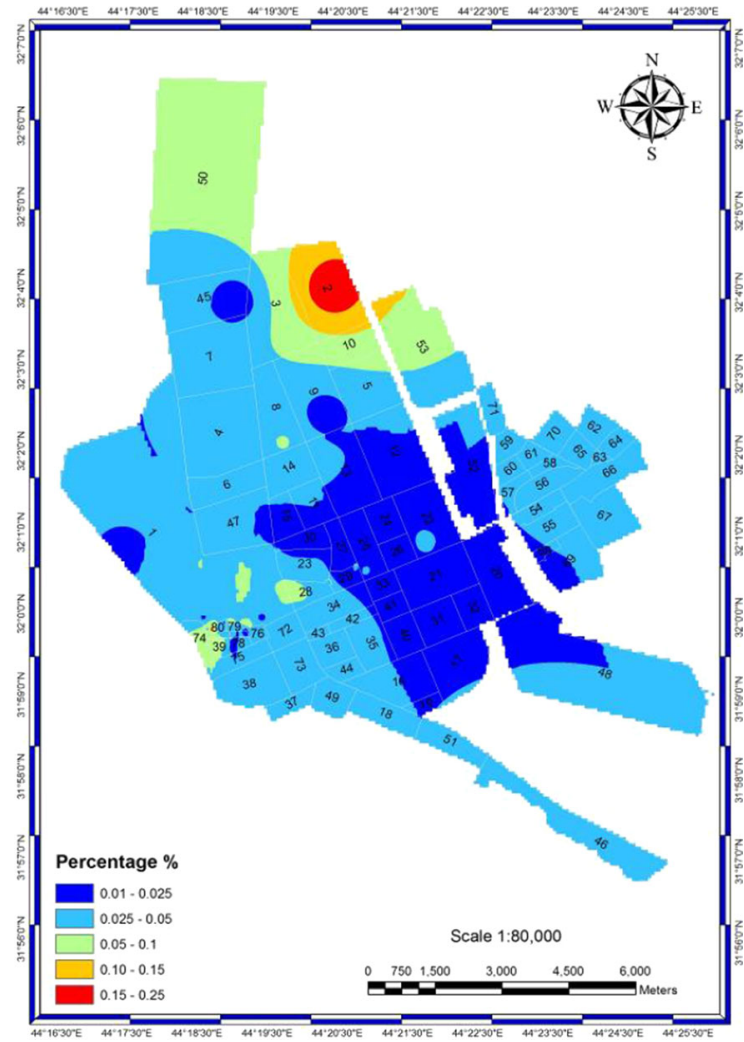

Fig. 10 Chloride concentration at depths 12-14 m

safe working with infrastructures during their lifecycle (Shahrour et al. 2007).

\section{Results and Discussion}

Figures 4, 5, 6, 7, 8, 9, 10, 11 and 12 show the produced maps for chloride concentrations.

From these maps it's found that chloride content in the soil of the study area range from -0.01 to $0.99 \%$

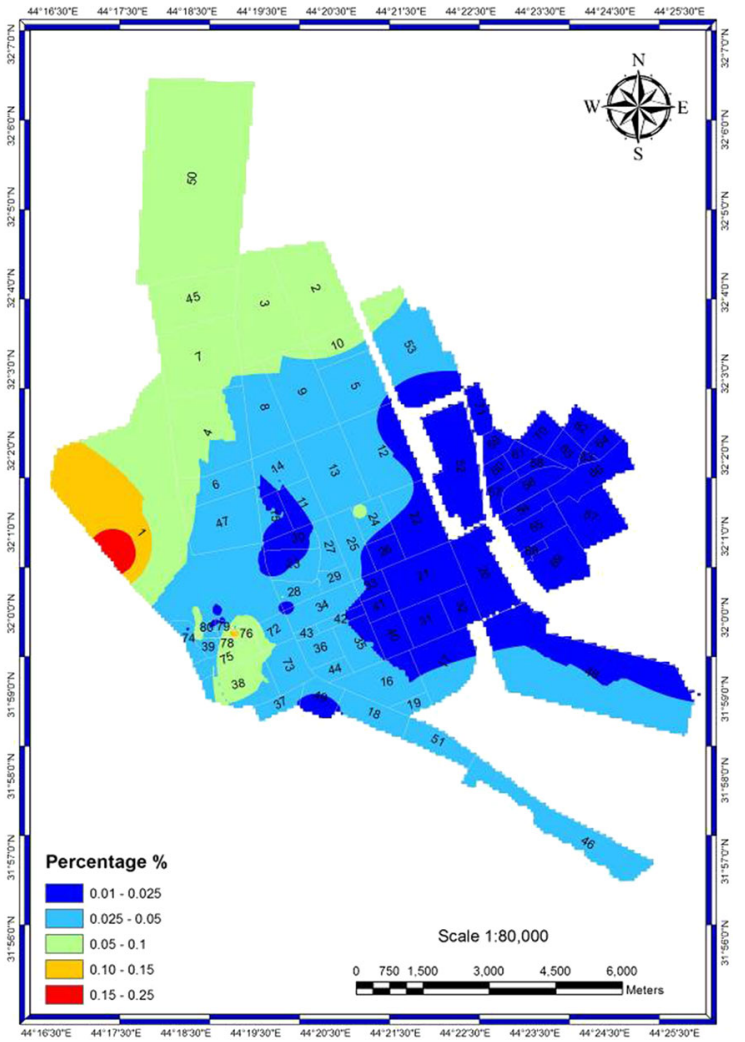

Fig. 11 Chloride concentration at depths 14-16 m

and with an average of 0.5 . The maximum value found in at depth 4-6 $\mathrm{m}$ while the minimum value found in location at depth $4-6,8-10$ and $14-16 \mathrm{~m}$. The maximum, minimum and average values for chloride content ranges in addition to the dangerous zone for each depth are listed in Table 2.

The high values of chloride salts content can be attributed to the rise in the groundwater levels in the region, As well as evaporation and drought factors. It is noted that the chloride content in most locations 


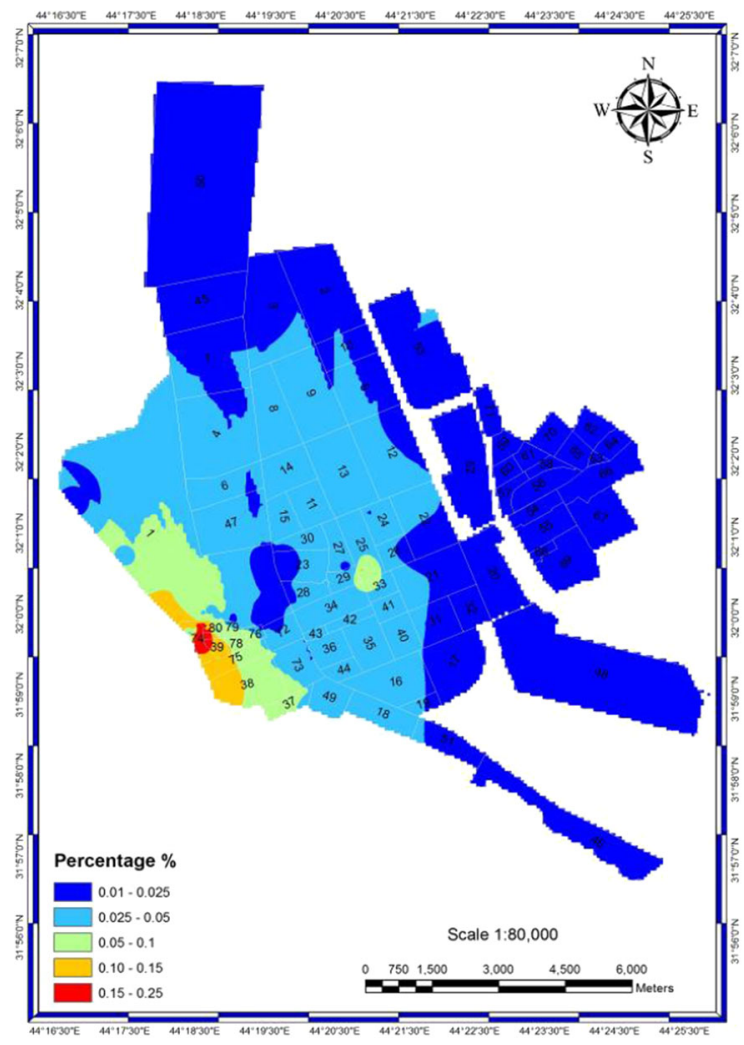

Fig. 12 Chloride concentration at depth $35 \mathrm{~ms}$ exceed the permissible value that effect on the soil engineering behavior which is $0.1 \%$, and that poses a danger to the foundations and reinforcing steel. Increasing chloride content leads to a decrease in Atterberg limits and an increase in the value of the maximum dry density. And affect the properties of compression resistance of concrete as the resistance decreases with increasing the chloride content, especially in the early age of concrete.

The produced maps for calcium carbonate content are listed in Figs. 13, 14, 15, 16, 17, 18, 19, 20 and 21.

From these maps it's found that calcium carbonate content in the soil of the study area range from -53 to 0.18 and with an average of 26.6. The maximum and the minimum values found at depth 4-6 $\mathrm{m}$. The maximum, minimum and average values for calcium carbonate content ranges in addition to the dangerous zone for each depth are listed in Table 3.

It is noted that the soil of the study area contains a high percentage of calcium carbonate in some locations. This may be due to the geological nature of Najaf, which contains large quantities of limestone, the dry climate contributes to the deposition of carbon materials from groundwater (Allen 2005) and its union with the calcium ion form calcium carbonate. In general, calcium carbonate content in most locations and for all depths has exceeded the permissible limit
Table 2 Chloride content ranges for all depths

\begin{tabular}{lllll}
\hline Depth & \multicolumn{3}{l}{ Chloride content $\%$} \\
\cline { 2 - 5 } & Min. & Max. & Average & Dangerous areas $>0.1 \%$ \\
\hline $0-2$ & 0.017 & $>0.025$ & 0.163 & $2,22,28,36,73,37$ \\
$2-4$ & 0.016 & $>0.25$ & 0.158 & $2,25,29,48,80$ \\
$4-6$ & 0.01 & $>0.25$ & 0.5 & $1,2,76,79,78,75,72,74,80,39,38$ \\
$6-8$ & 0.014 & 0.23 & 0.122 & $2,7,1,80,25,3,45$ \\
$8-10$ & 0.01 & $>0.25$ & 0.45 & $2,1,80,74,39,75,38,48,20,21,32,31,17,40,16,19$ \\
$10-12$ & 0.014 & 0.175 & 0.095 & 80 \\
$12-14$ & 0.017 & 0.21 & 0.114 & $2,3,10,53,80$ \\
$14-16$ & 0.01 & 0.195 & 0.102 & $1,76,79$ \\
$16-36$ & 0.017 & $>0.25$ & 0.4 & $25,1,74,80,39,78,75,38$ \\
\hline
\end{tabular}




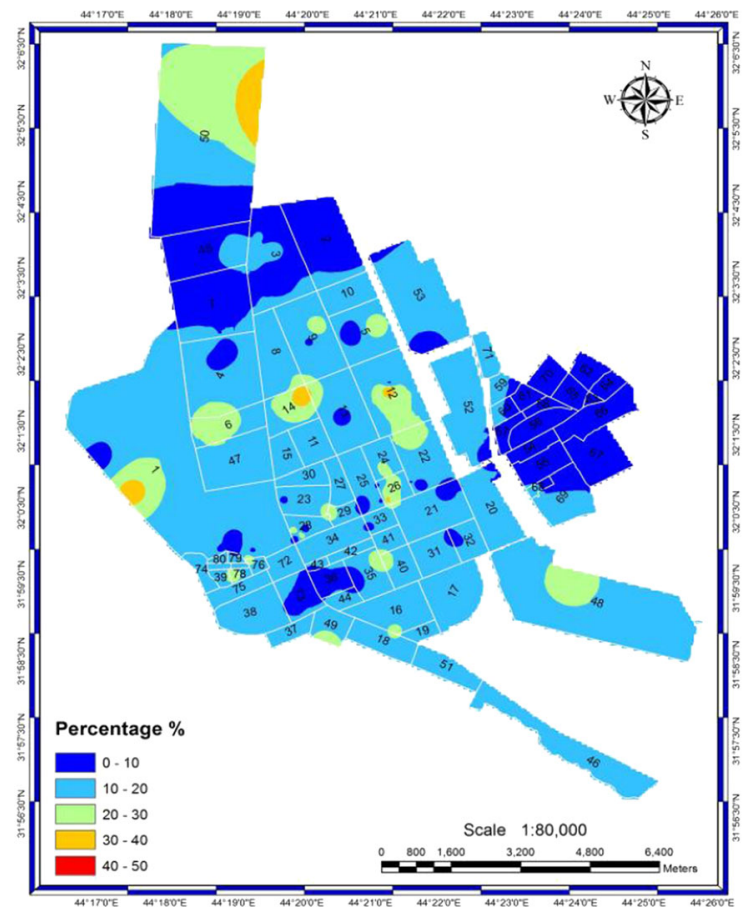

Fig. 13 Calcium carbonate content at depths 0-2 m

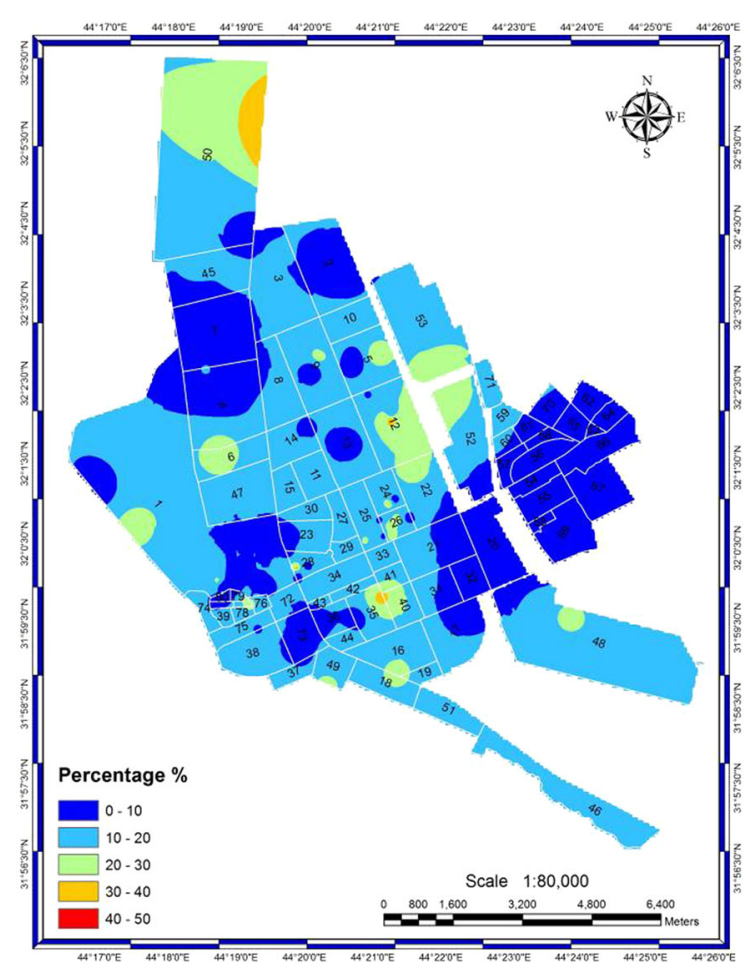

Fig. 14 Calcium carbonate content at depths 2-4 m

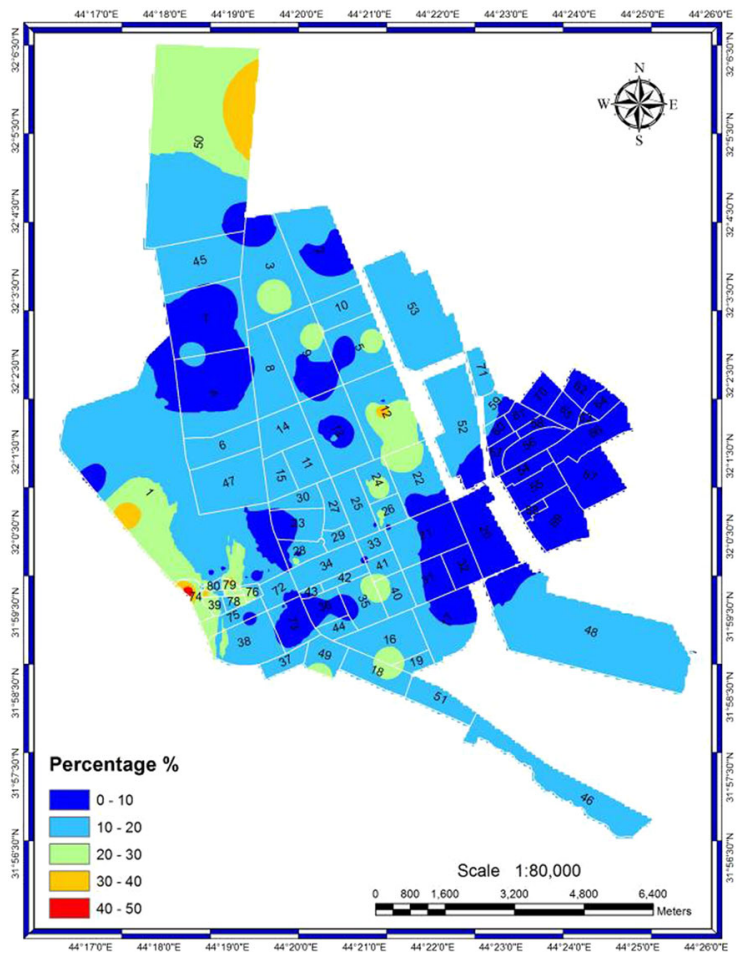

Fig. 15 Calcium carbonate content at depths 4-6 m

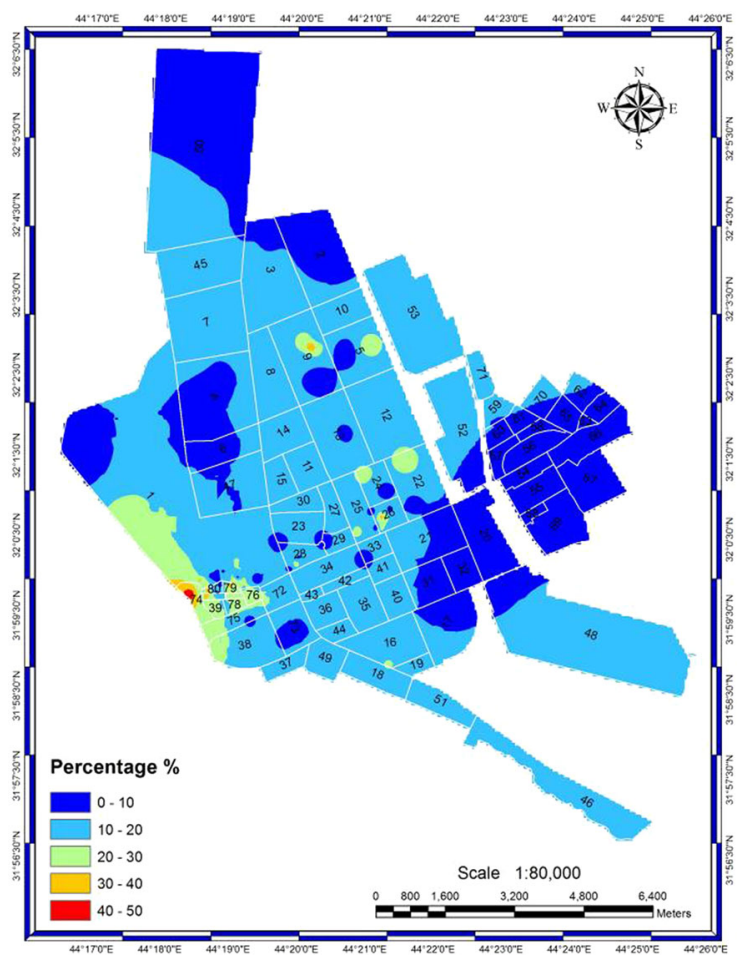

Fig. 16 Calcium carbonate content at depths 6-8 m 


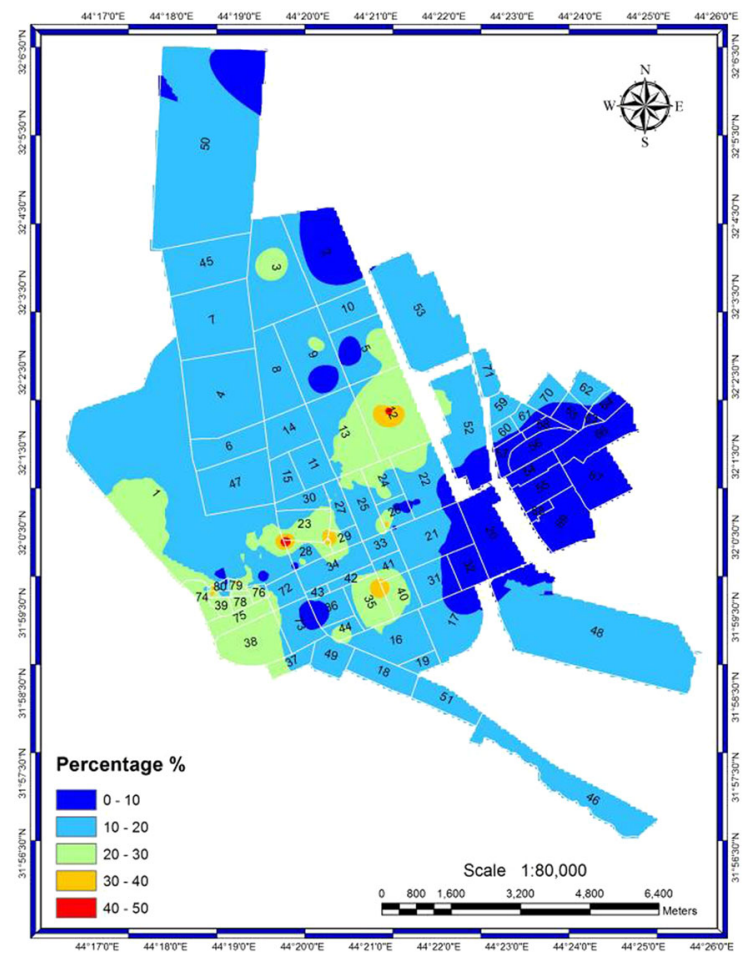

Fig. 17 Calcium carbonate content at depths 8-10 m

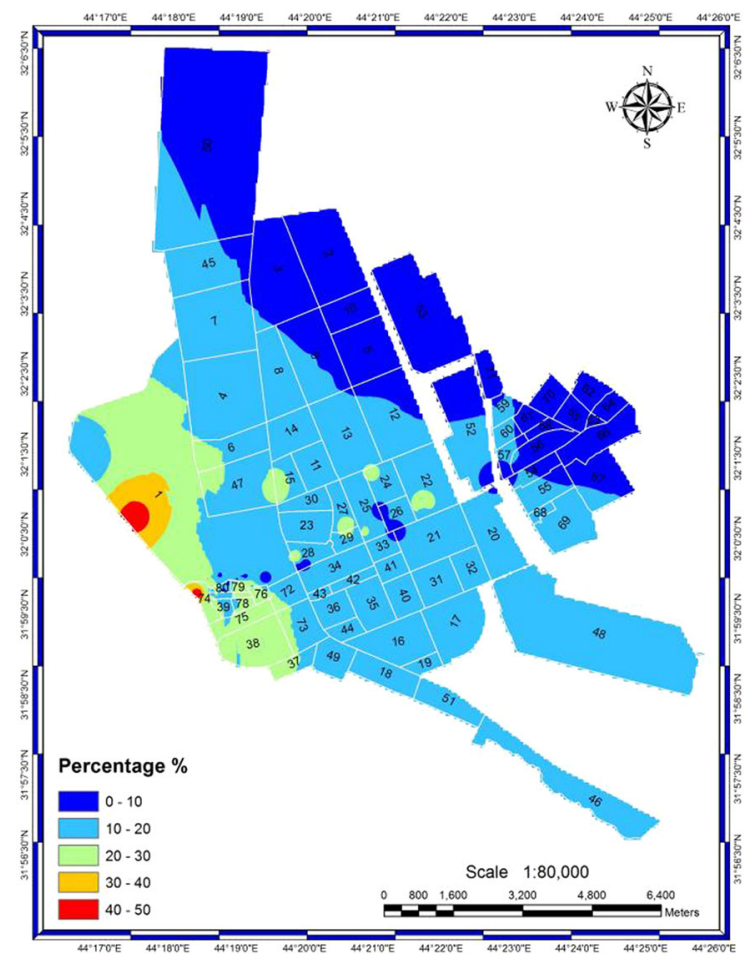

Fig. 18 Calcium carbonate content at depths 10-12 m

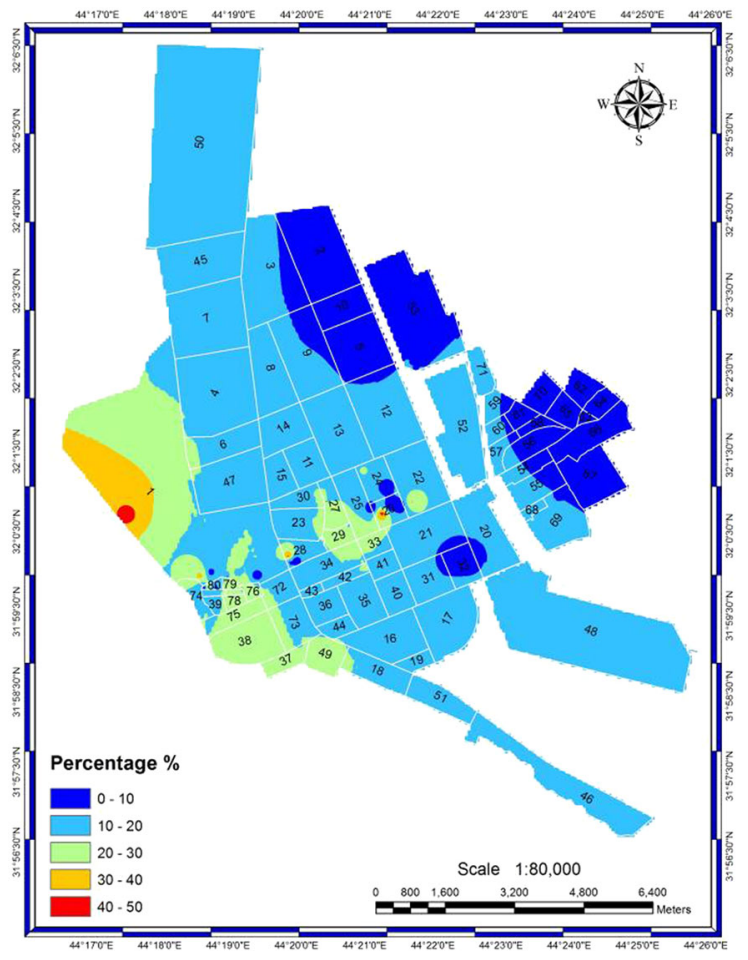

Fig. 19 Calcium carbonate content at depths 12-14 m

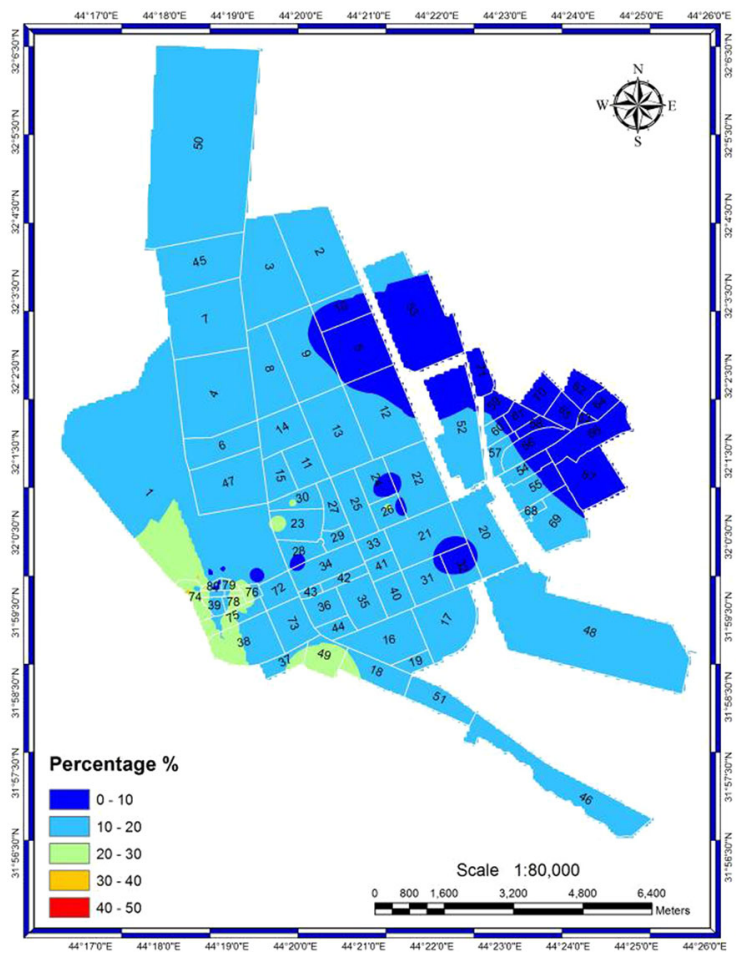

Fig. 20 Calcium carbonate content at depths 14-16 m 


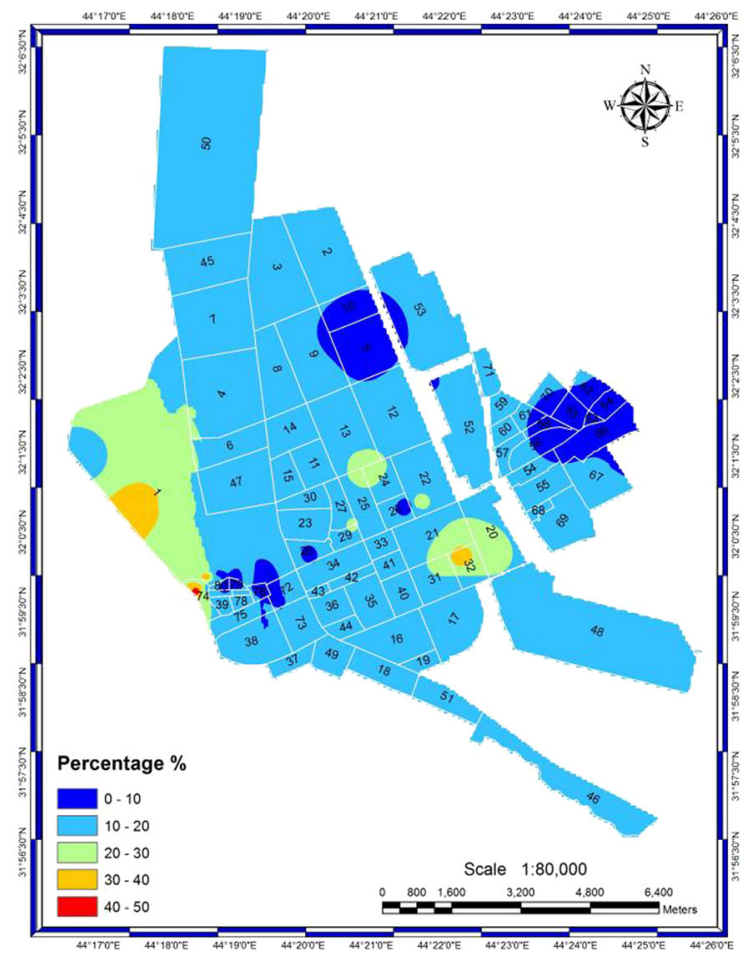

Fig. 21 Calcium carbonate content at depth $35 \mathrm{~m}$

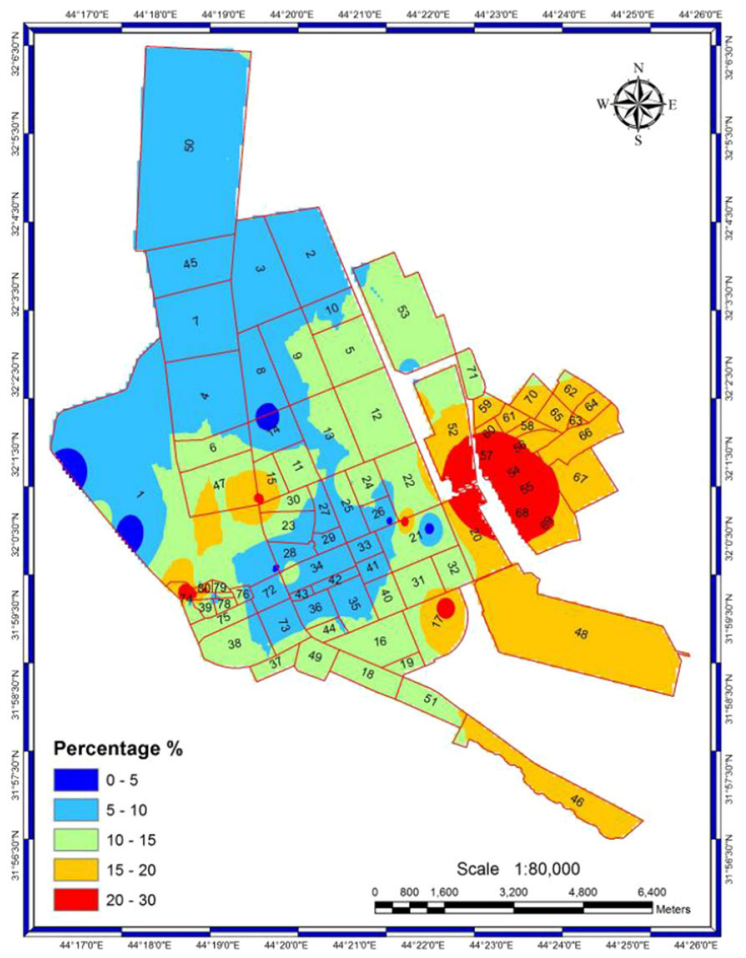

Fig. 22 TSS content at depths 0-2 m

Table 3 Calcium carbonate content ranges for all depths

\begin{tabular}{|c|c|c|c|c|}
\hline \multirow[t]{2}{*}{ Depth } & \multicolumn{4}{|c|}{ Calcium carbonate content $\%$} \\
\hline & Min. & Max. & Average & Location \\
\hline $0-2$ & 0.5 & 46.75 & 23.6 & $50,1,14,13,12,26$ \\
\hline $2-4$ & 0.5 & 46 & 23.25 & $1,28,26$ \\
\hline $4-6$ & 0.18 & 53 & 26.6 & $50,12,41,40,35,28$ \\
\hline $6-8$ & 0.5 & 52 & 26.25 & $50,12,1,74,80,79$ \\
\hline $8-10$ & 0.5 & 50 & 25.5 & $1,74,80,79,26,9$ \\
\hline $10-12$ & 0.5 & 52.5 & 26.5 & $12,80,1,23,28,29,41,40,35$ \\
\hline $12-14$ & 0.5 & 44 & 22.25 & $1,74,80$ \\
\hline $14-16$ & 0.5 & 35.5 & 18 & $1,74,80$ \\
\hline $16-36$ & 0.5 & 49.5 & 25 & $1,74,21,32$ \\
\hline
\end{tabular}


that affect the soil engineering behavior which is $30 \%$. So this pose a risk and lead to engineering problems such as cracks, buildings differential settlement and soil weakening. As for total soluble salts (TSS) the produced maps included in Figs. 22, 23, 24, 25, 26, 27 , 28 and 29.

\section{Conclusions}

1. Geographic Information System a helpful way to predict soil properties in non-Spatial data locations.

2. Geotechnical maps created by this work illustrate a powerful database and visual display of the data.

3. Using such maps help saving time, expenses and potentials.

4. Eventual maps created by this research will be used as a guidance for engineers and decisions makers to decide the safety of the site soil to build any project and type of suitable treatment needed.

5. The chloride content in the soil of the study area range from -0.01 to $0.99 \%$ and with an average of 0.5 . The maximum value found in at depth

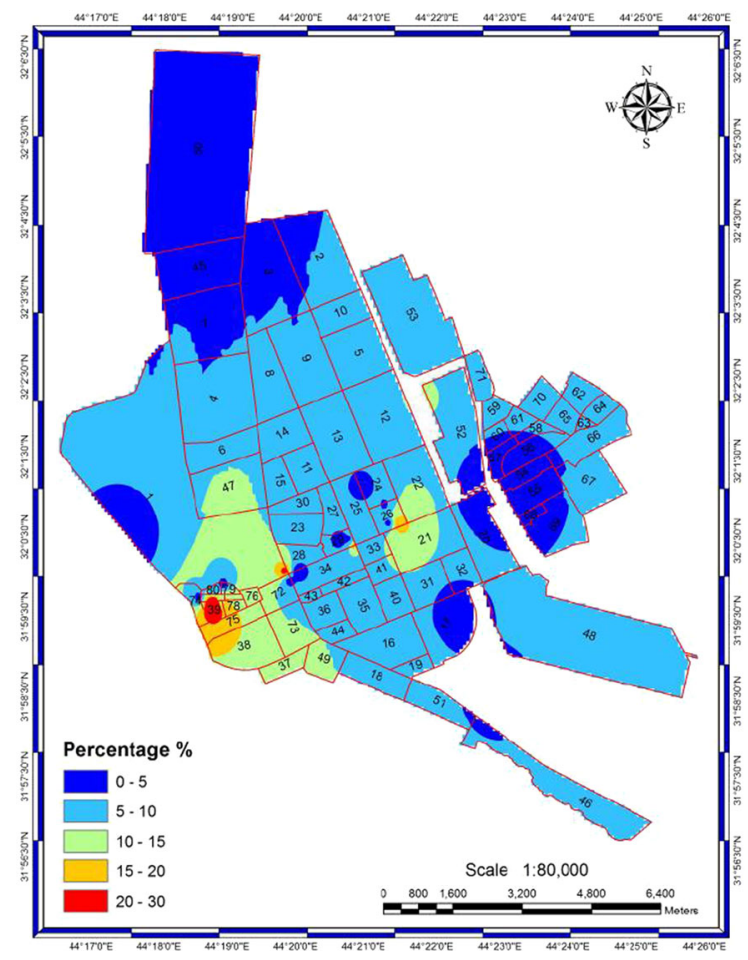

Fig. 23 TSS content at depths 2-4 m

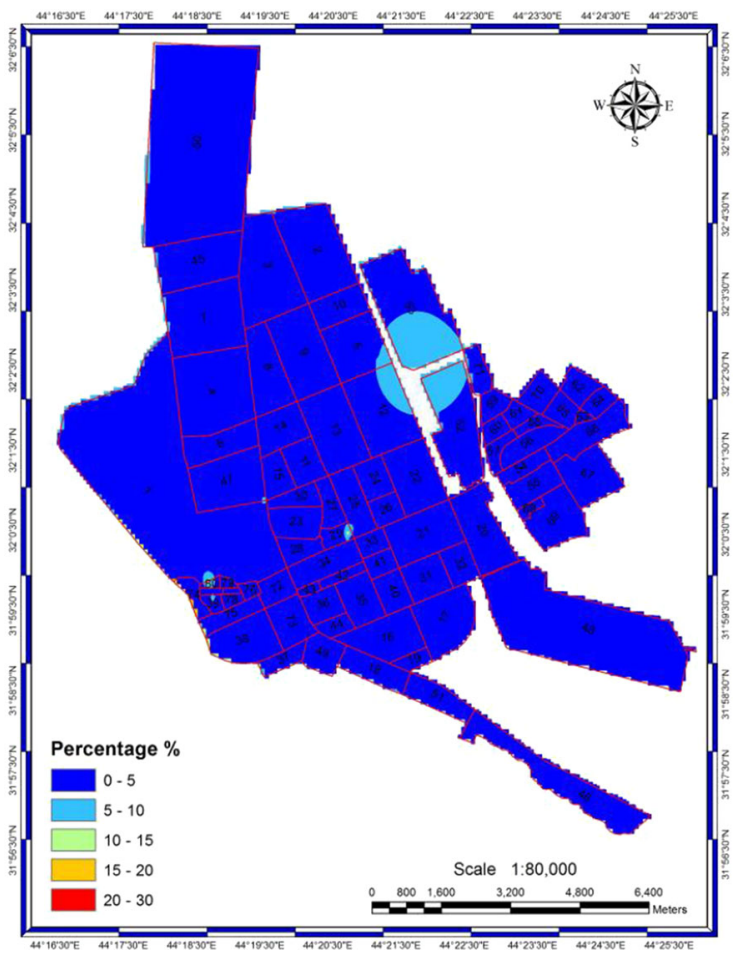

Fig. 24 TSS content at depths 4-6 m

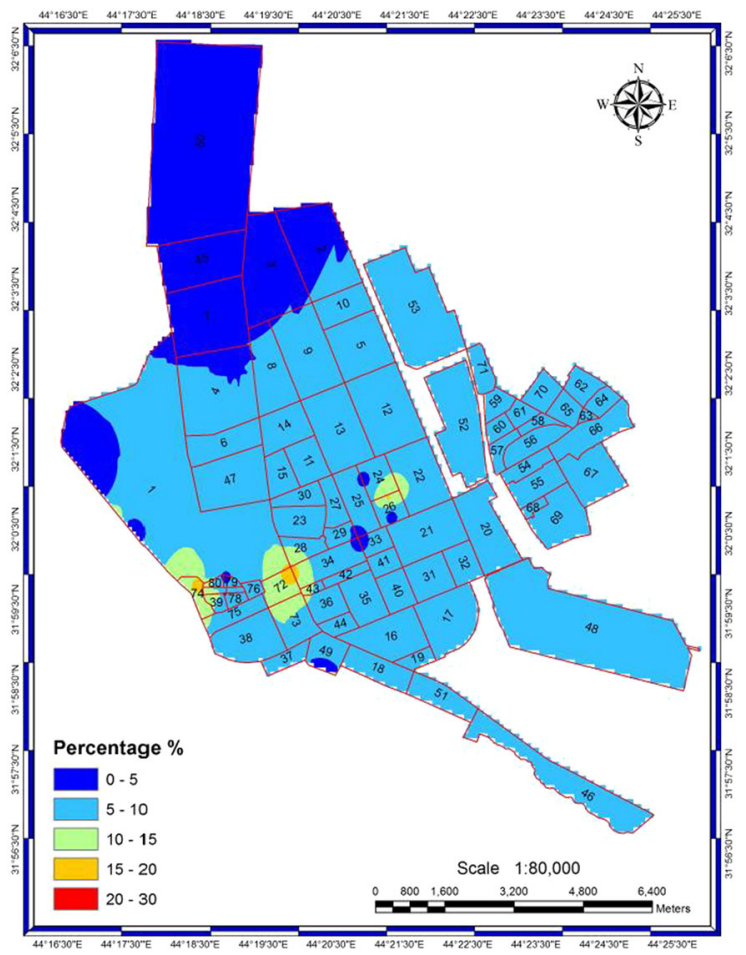

Fig. 25 TSS content at depths 6-8 m 


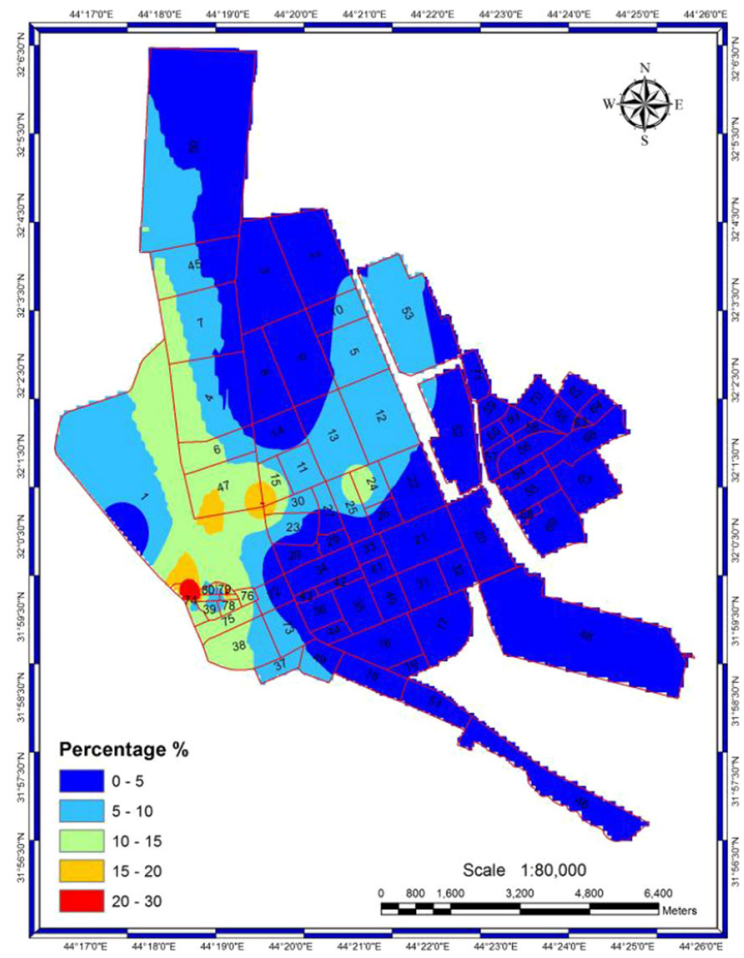

Fig. 26 TSS content at depths $8-10 \mathrm{~m}$

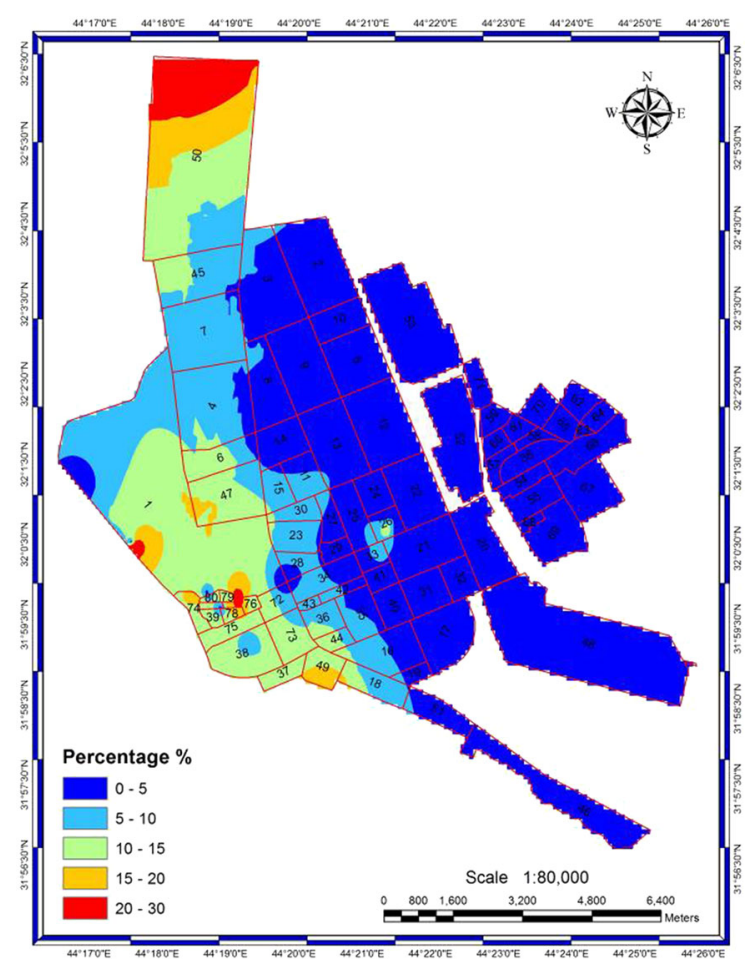

Fig. 27 TSS content at depths 10-12 m

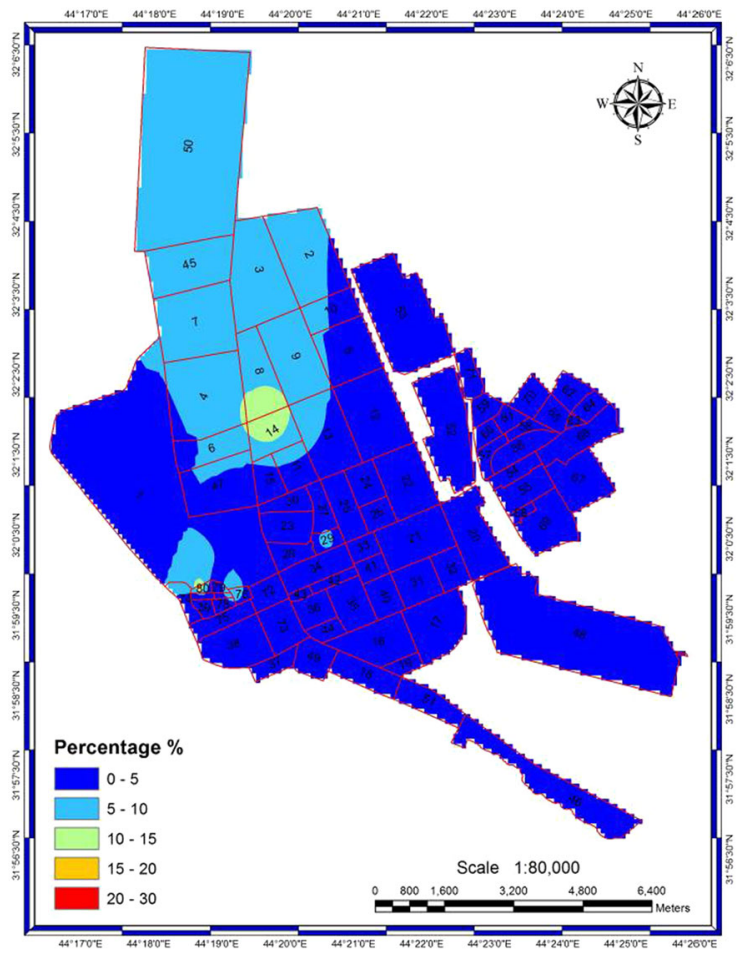

Fig. 28 TSS content at depths $12-14 \mathrm{~m}$

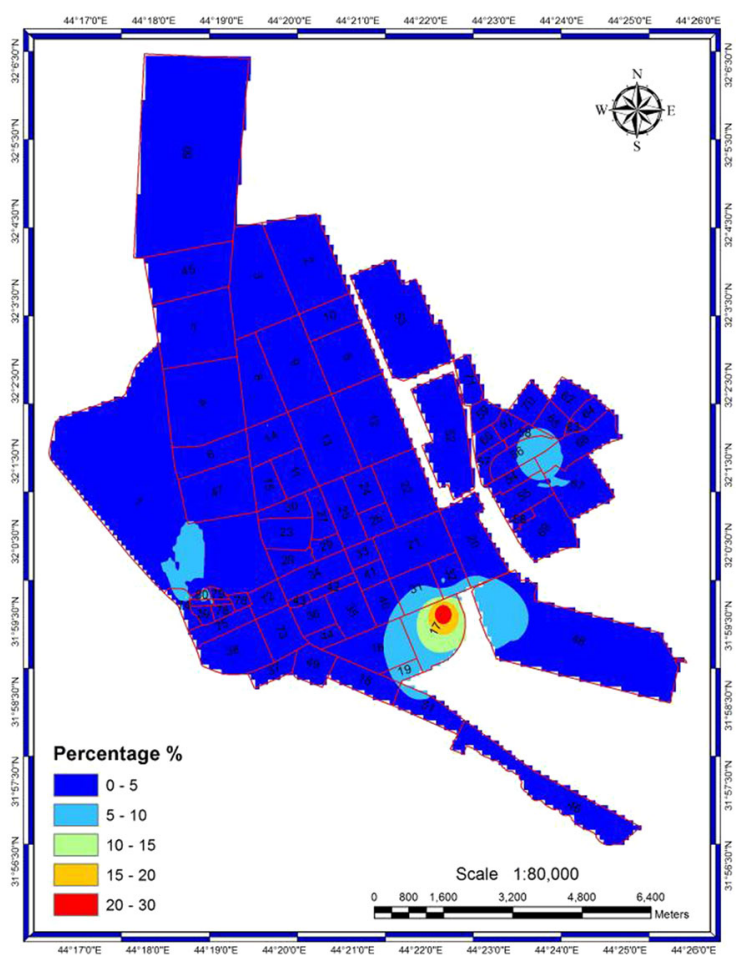

Fig. 29 TSS content at depths $12-36 \mathrm{~m}$ 
4-6 $\mathrm{m}$ while the minimum value found in location at depth 4-6, 8-10 and 14-16 m.

6. The chloride content in most of An-Najaf province has exceeded the permissible limit in most locations and for all and this required taking percussions to protect foundations.

7. Calcium Carbonate content in the soil of the study area range from -0.18 to 53 and with an average of 26.6. The maximum and the minimum values found at depth 4-6 m.

8. Calcium Carbonate content is within the permissible limits in most locations except in some locations.

9. The results show that the salinity of soil in the governorate is true but not absolute because salinity is unequal for all locations and depths. This salinity is liable to increase and decrease over time according to the geological and environmental factors affecting it.

Open Access This article is distributed under the terms of the Creative Commons Attribution 4.0 International License (http:// creativecommons.org/licenses/by/4.0/), which permits unrestricted use, distribution, and reproduction in any medium, provided you give appropriate credit to the original author(s) and the source, provide a link to the Creative Commons license, and indicate if changes were made.

\section{Appendix: Boreholes Coordinates of Wells}

\begin{tabular}{|c|c|c|c|c|c|c|c|c|c|c|c|}
\hline No. & $\begin{array}{l}\text { Borehole } \\
\text { codes }\end{array}$ & Long. & Latt. & No. & $\begin{array}{l}\text { Borehole } \\
\text { codes }\end{array}$ & Long. & Latt. & No. & $\begin{array}{l}\text { Borehole } \\
\text { codes }\end{array}$ & Long. & Latt. \\
\hline 1 & 1001 & $4420^{\prime} 56^{\prime \prime}$ & $320^{\prime} 6^{\prime \prime}$ & 56 & 2019 & $4418^{\prime} 48^{\prime \prime}$ & $3159^{\prime} 43^{\prime \prime}$ & 111 & 1033 & $4420^{\prime} 3^{\prime \prime}$ & $3159^{\prime} 37^{\prime}$ \\
\hline 2 & 2001 & $4420^{\prime} 56^{\prime \prime}$ & $320^{\prime} 6^{\prime \prime}$ & 57 & 3019 & $4418^{\prime} 41^{\prime \prime}$ & $3159^{\prime} 44^{\prime \prime}$ & 112 & 2033 & $4420^{\prime} 3^{\prime \prime}$ & $3159^{\prime} 37^{\prime}$ \\
\hline 3 & 1002 & $4420^{\prime} 51^{\prime \prime}$ & $320^{\prime} 25^{\prime \prime}$ & 58 & 1020 & $4419^{\prime} 22^{\prime \prime}$ & $3159^{\prime} 49^{\prime \prime}$ & 113 & 1034 & $4422^{\prime} 50^{\prime \prime}$ & $3158^{\prime} 36^{\prime}$ \\
\hline 4 & 2002 & $4420^{\prime} 50^{\prime \prime}$ & $320^{\prime} 25^{\prime \prime}$ & 59 & 2020 & $4419^{\prime} 22^{\prime \prime}$ & $3159^{\prime} 49^{\prime \prime}$ & 114 & 2034 & $4422^{\prime} 52^{\prime \prime}$ & $3158^{\prime} 40^{\prime}$ \\
\hline 5 & 3002 & $4420^{\prime} 49^{\prime \prime}$ & $320^{\prime} 25^{\prime \prime}$ & 60 & 1021 & $4418^{\prime} 38^{\prime \prime}$ & $3159^{\prime} 41^{\prime \prime}$ & 115 & 3034 & $4422^{\prime} 53^{\prime \prime}$ & $3158^{\prime} 37^{\prime}$ \\
\hline 6 & 1003 & $4420^{\prime} 40^{\prime \prime}$ & $322^{\prime} 39^{\prime \prime}$ & 61 & 2021 & $4418^{\prime} 38^{\prime \prime}$ & $3159^{\prime} 41^{\prime \prime}$ & 116 & 4034 & $4422^{\prime} 54^{\prime \prime}$ & $3158^{\prime} 36^{\prime}$ \\
\hline 7 & 2003 & $4420^{\prime} 40^{\prime \prime}$ & $322^{\prime} 39^{\prime \prime}$ & 62 & 1022 & $4421^{\prime} 6^{\prime \prime}$ & $3159^{\prime} 26^{\prime \prime}$ & 117 & 5034 & $4422^{\prime} 57^{\prime \prime}$ & $3158^{\prime} 36^{\prime}$ \\
\hline 8 & 1004 & $4419^{\prime} 43^{\prime \prime}$ & $320^{\prime} 15^{\prime \prime}$ & 63 & 2022 & $4421^{\prime} 6^{\prime \prime}$ & $3159^{\prime} 29^{\prime \prime}$ & 118 & 6034 & $4422^{\prime} 55^{\prime \prime}$ & $3158^{\prime} 33^{\prime \prime}$ \\
\hline 9 & 2004 & $4419^{\prime} 42^{\prime \prime}$ & $320^{\prime} 17^{\prime \prime}$ & 64 & 1023 & $4422^{\prime} 48^{\prime \prime}$ & $3159^{\prime} 28^{\prime \prime}$ & 119 & 1035 & $4420^{\prime} 15^{\prime \prime}$ & $322^{\prime} 19^{\prime \prime}$ \\
\hline 10 & 1005 & $4420^{\prime} 0^{\prime \prime}$ & $320^{\prime} 44^{\prime \prime}$ & 65 & 2023 & $4423^{\prime} 49^{\prime \prime}$ & $3159^{\prime} 28^{\prime \prime}$ & 120 & 2035 & $4420^{\prime} 14^{\prime \prime}$ & $322^{\prime} 19^{\prime \prime}$ \\
\hline 11 & 2005 & $4419^{\prime} 57^{\prime \prime}$ & $320^{\prime} 47^{\prime \prime}$ & 66 & 3023 & $4424^{\prime} 15^{\prime \prime}$ & $3158^{\prime} 51^{\prime \prime}$ & 121 & 3035 & $4420^{\prime} 15^{\prime \prime}$ & $322^{\prime} 19^{\prime \prime}$ \\
\hline 12 & 1006 & $4419^{\prime} 13^{\prime \prime}$ & $324^{\prime} 11^{\prime \prime}$ & 67 & 4023 & $4423^{\prime} 28^{\prime \prime}$ & $3159^{\prime} 56^{\prime \prime}$ & 122 & 1036 & $4418^{\prime} 29^{\prime \prime}$ & $3159^{\prime} 41^{\prime}$ \\
\hline 13 & 2006 & $4419^{\prime} 14^{\prime \prime}$ & $324^{\prime} 10^{\prime \prime}$ & 68 & 5023 & $4423^{\prime} 53^{\prime \prime}$ & $3159^{\prime} 29^{\prime \prime}$ & 123 & 2036 & $4418^{\prime} 29^{\prime \prime}$ & $3159^{\prime} 39^{\prime}$ \\
\hline 14 & 1007 & $4418^{\prime} 34^{\prime \prime}$ & $3159^{\prime} 45^{\prime \prime}$ & 69 & 6023 & $4423^{\prime} 57^{\prime \prime}$ & $3159^{\prime} 28^{\prime \prime}$ & 124 & 3036 & $4418^{\prime} 28^{\prime \prime}$ & $3159^{\prime} 40^{\prime}$ \\
\hline 15 & 2007 & $4418^{\prime} 33^{\prime \prime}$ & $3159^{\prime} 44^{\prime \prime}$ & 70 & 7023 & $4423^{\prime} 51^{\prime \prime}$ & $3159^{\prime} 29^{\prime \prime}$ & 125 & 4036 & $4418^{\prime} 28^{\prime \prime}$ & $3159^{\prime} 41^{\prime \prime}$ \\
\hline 16 & 3007 & $4418^{\prime} 33^{\prime \prime}$ & $3159^{\prime} 45^{\prime \prime}$ & 71 & 8023 & $4423^{\prime} 51^{\prime \prime}$ & $3159^{\prime} 29^{\prime \prime}$ & 126 & 5036 & $4418^{\prime} 27^{\prime \prime}$ & $3159^{\prime} 41^{\prime \prime}$ \\
\hline 17 & 1008 & $4418^{\prime} 39^{\prime \prime}$ & $3159^{\prime} 28^{\prime \prime}$ & 72 & 9023 & $4423^{\prime} 50^{\prime \prime}$ & $3159^{\prime} 29^{\prime \prime}$ & 127 & 1037 & $4418^{\prime} 47^{\prime \prime}$ & $3159^{\prime} 42^{\prime \prime}$ \\
\hline 18 & 2008 & $4419^{\prime} 4^{\prime \prime}$ & $3159^{\prime} 29^{\prime \prime}$ & 73 & 10023 & $4423^{\prime} 55^{\prime \prime}$ & $3159^{\prime} 28^{\prime \prime}$ & 128 & 2037 & $4418^{\prime} 48^{\prime \prime}$ & $3159^{\prime} 42^{\prime}$ \\
\hline 19 & 1009 & $4417^{\prime} 32^{\prime \prime}$ & $320^{\prime} 25^{\prime \prime}$ & 74 & 11023 & $4423^{\prime} 54^{\prime \prime}$ & $3159^{\prime} 29^{\prime \prime}$ & 129 & 1038 & $4418^{\prime} 36^{\prime \prime}$ & $3159^{\prime} 41^{\prime \prime}$ \\
\hline 20 & 2009 & $4417^{\prime} 36^{\prime \prime}$ & $320^{\prime} 29^{\prime \prime}$ & 75 & 12023 & $4423^{\prime} 22^{\prime \prime}$ & $3159^{\prime} 41^{\prime \prime}$ & 130 & 2038 & $4418^{\prime} 36^{\prime \prime}$ & $3159^{\prime} 41^{\prime \prime}$ \\
\hline 21 & 3009 & $4417^{\prime} 34^{\prime \prime}$ & $320^{\prime} 31^{\prime \prime}$ & 76 & 1024 & $4421^{\prime} 3^{\prime \prime}$ & $320^{\prime} 40^{\prime \prime}$ & 131 & 1039 & $4418^{\prime} 43^{\prime \prime}$ & $3159^{\prime} 42^{\prime}$ \\
\hline 22 & 4009 & $4417^{\prime} 29^{\prime \prime}$ & $320^{\prime} 28^{\prime \prime}$ & 77 & 2024 & $4421^{\prime} 3^{\prime \prime}$ & $320^{\prime} 39^{\prime \prime}$ & 132 & 2039 & $4418^{\prime} 44^{\prime \prime}$ & $3159^{\prime} 41^{\prime}$ \\
\hline 23 & 5009 & $4417^{\prime} 23^{\prime \prime}$ & $320^{\prime} 30^{\prime \prime}$ & 78 & 1025 & $4418^{\prime} 33^{\prime \prime}$ & $3159^{\prime} 49^{\prime \prime}$ & 133 & 1040 & $4418^{\prime} 57^{\prime \prime}$ & $323^{\prime} 42^{\prime \prime}$ \\
\hline 24 & 6009 & $4417^{\prime} 29^{\prime \prime}$ & $320^{\prime} 37^{\prime \prime}$ & 79 & 2025 & $4418^{\prime} 33^{\prime \prime}$ & $3159^{\prime} 49^{\prime \prime}$ & 134 & 2040 & $4418^{\prime} 59^{\prime \prime}$ & $323^{\prime} 42^{\prime \prime}$ \\
\hline 25 & 7009 & $4417^{\prime} 21^{\prime \prime}$ & $320^{\prime} 32^{\prime \prime}$ & 80 & 3025 & $4418^{\prime} 32^{\prime \prime}$ & $3159^{\prime} 48^{\prime \prime}$ & 135 & 3040 & $4419^{\prime} 0^{\prime \prime}$ & $323^{\prime} 43^{\prime \prime}$ \\
\hline 26 & 8009 & $4417^{\prime} 19^{\prime \prime}$ & $320^{\prime} 34^{\prime \prime}$ & 81 & 4025 & $4418^{\prime} 32^{\prime \prime}$ & $3159^{\prime} 48^{\prime \prime}$ & 136 & 4040 & $4418^{\prime} 59^{\prime \prime}$ & $323^{\prime} 43^{\prime \prime}$ \\
\hline 27 & 9009 & $4417^{\prime} 21^{\prime \prime}$ & $320^{\prime} 37^{\prime \prime}$ & 82 & 1026 & $4416^{\prime} 46^{\prime \prime}$ & $321^{\prime} 12^{\prime \prime}$ & 137 & 1041 & $4421^{\prime} 19^{\prime \prime}$ & $320^{\prime} 41^{\prime \prime}$ \\
\hline 28 & 10009 & $4417^{\prime} 15^{\prime \prime}$ & $320^{\prime} 37^{\prime \prime}$ & 83 & 2026 & $4416^{\prime} 41^{\prime \prime}$ & $321^{\prime} 12^{\prime \prime}$ & 138 & 2041 & $4421^{\prime} 20^{\prime \prime}$ & $320^{\prime} 41^{\prime \prime}$ \\
\hline 29 & 1010 & $4418^{\prime} 24^{\prime \prime}$ & $323^{\prime} 55^{\prime \prime}$ & 84 & 1027 & $4419^{\prime} 52^{\prime \prime}$ & $3159^{\prime} 59^{\prime \prime}$ & 139 & 3041 & $4421^{\prime} 18^{\prime \prime}$ & $320^{\prime} 41^{\prime \prime}$ \\
\hline
\end{tabular}


continued

\begin{tabular}{|c|c|c|c|c|c|c|c|c|c|c|c|}
\hline No. & $\begin{array}{l}\text { Borehole } \\
\text { codes }\end{array}$ & Long. & Latt. & No. & $\begin{array}{l}\text { Borehole } \\
\text { codes }\end{array}$ & Long. & Latt. & No. & $\begin{array}{l}\text { Borehole } \\
\text { codes }\end{array}$ & Long. & Latt. \\
\hline 30 & 2010 & $4418^{\prime} 25^{\prime \prime}$ & $323^{\prime} 54^{\prime \prime}$ & 85 & 2027 & $4419^{\prime} 52^{\prime \prime}$ & $3159^{\prime} 59^{\prime \prime}$ & 140 & 4041 & $4421^{\prime} 20^{\prime \prime}$ & $320^{\prime} 42^{\prime \prime}$ \\
\hline 31 & 1011 & $4422^{\prime} 40^{\prime \prime}$ & $321^{\prime} 4^{\prime \prime}$ & 86 & 1028 & $4419^{\prime} 11^{\prime \prime}$ & $3159^{\prime} 42^{\prime \prime}$ & 141 & 1042 & $4419^{\prime} 31^{\prime \prime}$ & $323^{\prime} 44^{\prime \prime}$ \\
\hline 32 & 2011 & $4422^{\prime} 44^{\prime \prime}$ & $321^{\prime} 3^{\prime \prime}$ & 87 & 2028 & $4419^{\prime} 11^{\prime \prime}$ & $3159^{\prime} 42^{\prime \prime}$ & 142 & 1043 & $4421^{\prime} 12^{\prime \prime}$ & $321^{\prime} 59^{\prime \prime}$ \\
\hline 33 & 3011 & $4422^{\prime} 42^{\prime \prime}$ & $321^{\prime} 5^{\prime \prime}$ & 88 & 1029 & $4419^{\prime} 56^{\prime \prime}$ & $320^{\prime} 2^{\prime \prime}$ & 143 & 2043 & $4421^{\prime} 13^{\prime \prime}$ & $322^{\prime} 0^{\prime \prime}$ \\
\hline 34 & 1012 & $4418^{\prime} 37^{\prime \prime}$ & $3159^{\prime} 40^{\prime \prime}$ & 89 & 2029 & $4419^{\prime} 54^{\prime \prime}$ & $320^{\prime} 2^{\prime \prime}$ & 144 & 3043 & $4421^{\prime} 13^{\prime \prime}$ & $321^{\prime} 59^{\prime \prime}$ \\
\hline 35 & 2012 & $4418^{\prime} 36^{\prime \prime}$ & $3159^{\prime} 40^{\prime \prime}$ & 90 & 3029 & $4419^{\prime} 56^{\prime \prime}$ & $320^{\prime} 3^{\prime \prime}$ & 145 & 1044 & $4420^{\prime} 18^{\prime \prime}$ & $323^{\prime} 59^{\prime \prime}$ \\
\hline 36 & 1013 & $4419^{\prime} 53^{\prime \prime}$ & $320^{\prime} 44^{\prime \prime}$ & 91 & 4029 & $4419^{\prime} 56^{\prime \prime}$ & $3159^{\prime} 58^{\prime \prime}$ & 146 & 2044 & $4420^{\prime} 16^{\prime \prime}$ & $324^{\prime} 0^{\prime \prime}$ \\
\hline 37 & 2013 & $4419^{\prime} 53^{\prime \prime}$ & $320^{\prime} 43^{\prime \prime}$ & 92 & 5029 & $4419^{\prime} 57^{\prime \prime}$ & $3159^{\prime} 59^{\prime \prime}$ & 147 & 1045 & $4418^{\prime} 47^{\prime \prime}$ & $320^{\prime} 29^{\prime \prime}$ \\
\hline 38 & 3013 & $4419^{\prime} 56^{\prime \prime}$ & $320^{\prime} 43^{\prime \prime}$ & 93 & 6029 & $4419^{\prime} 59^{\prime \prime}$ & $320^{\prime} 2^{\prime \prime}$ & 148 & 2045 & $4418^{\prime} 52^{\prime \prime}$ & $320^{\prime} 37^{\prime \prime}$ \\
\hline 39 & 4013 & $4419^{\prime} 56^{\prime \prime}$ & $320^{\prime} 44^{\prime \prime}$ & 94 & 7029 & $4419^{\prime} 59^{\prime \prime}$ & $320^{\prime} 0^{\prime \prime}$ & 149 & 3045 & $4419^{\prime} 1^{\prime \prime}$ & $320^{\prime} 32^{\prime \prime}$ \\
\hline 40 & 5013 & $4419^{\prime} 54^{\prime \prime}$ & $320^{\prime} 44^{\prime \prime}$ & 95 & 1030 & $4419^{\prime} 48^{\prime \prime}$ & $320^{\prime} 4^{\prime \prime}$ & 150 & 4045 & $4418^{\prime} 34^{\prime \prime}$ & $320^{\prime} 34^{\prime \prime}$ \\
\hline 41 & 6013 & $4419^{\prime} 54^{\prime \prime}$ & $320^{\prime} 45^{\prime \prime}$ & 96 & 2030 & $4419^{\prime} 51^{\prime \prime}$ & $320^{\prime} 2^{\prime \prime}$ & 151 & 5045 & $4418^{\prime} 38^{\prime \prime}$ & $320^{\prime} 37^{\prime \prime}$ \\
\hline 42 & 7013 & $4420^{\prime} 2^{\prime \prime}$ & $320^{\prime} 44^{\prime \prime}$ & 97 & 3030 & $4419^{\prime} 51^{\prime \prime}$ & $320^{\prime} 4^{\prime \prime}$ & 152 & 6045 & $4418^{\prime} 41^{\prime \prime}$ & $320^{\prime} 37^{\prime \prime}$ \\
\hline 43 & 1014 & $4421^{\prime} 17^{\prime \prime}$ & $320^{\prime} 27^{\prime \prime}$ & 98 & 4030 & $4419^{\prime} 50^{\prime \prime}$ & $320^{\prime} 5^{\prime \prime}$ & 153 & 7045 & $4418^{\prime} 37^{\prime \prime}$ & $320^{\prime} 41^{\prime \prime}$ \\
\hline 44 & 2014 & $4421^{\prime} 18^{\prime \prime}$ & $320^{\prime} 26^{\prime \prime}$ & 99 & 1031 & $4421^{\prime} 22^{\prime \prime}$ & $3158^{\prime} 42^{\prime \prime}$ & 154 & 1046 & $4421^{\prime} 19^{\prime \prime}$ & $320^{\prime} 41^{\prime \prime}$ \\
\hline 45 & 1015 & $4421^{\prime} 29^{\prime \prime}$ & $320^{\prime} 48^{\prime \prime}$ & 100 & 2031 & $4421^{\prime} 16^{\prime \prime}$ & $3158^{\prime} 46^{\prime \prime}$ & 155 & 2046 & $4421^{\prime} 20^{\prime \prime}$ & $320^{\prime} 41^{\prime \prime}$ \\
\hline 46 & 2015 & $4421^{\prime} 14^{\prime \prime}$ & $320^{\prime} 57^{\prime \prime}$ & 101 & 3031 & $4421^{\prime} 10^{\prime \prime}$ & $3158^{\prime} 47^{\prime \prime}$ & 156 & 3046 & $4421^{\prime} 18^{\prime \prime}$ & $320^{\prime} 41^{\prime \prime}$ \\
\hline 47 & 1016 & $4421^{\prime} 27^{\prime \prime}$ & $321^{\prime} 4^{\prime \prime}$ & 102 & 4031 & $4421^{\prime} 20^{\prime \prime}$ & $3158^{\prime} 45^{\prime \prime}$ & 157 & 4046 & $4421^{\prime} 20^{\prime \prime}$ & $320^{\prime} 42^{\prime \prime}$ \\
\hline 48 & 2016 & $4421^{\prime} 30^{\prime \prime}$ & $320^{\prime} 43^{\prime \prime}$ & 103 & 5031 & $4421^{\prime} 13^{\prime \prime}$ & $3158^{\prime} 48^{\prime \prime}$ & 158 & 5046 & $4418^{\prime} 49^{\prime \prime}$ & $3159^{\prime} 43^{\prime \prime}$ \\
\hline 49 & 1017 & $4423^{\prime} 29^{\prime \prime}$ & $3159^{\prime} 34^{\prime \prime}$ & 104 & 6031 & $4421^{\prime} 26^{\prime \prime}$ & $3158^{\prime} 46^{\prime \prime}$ & 159 & 6046 & $4418^{\prime} 45^{\prime \prime}$ & $3159^{\prime} 43^{\prime \prime}$ \\
\hline 50 & 2017 & $4423^{\prime} 43^{\prime \prime}$ & $3159^{\prime} 30^{\prime \prime}$ & 105 & 7031 & $4421^{\prime} 17^{\prime \prime}$ & $3158^{\prime} 48^{\prime \prime}$ & 160 & 7046 & $4418^{\prime} 40^{\prime \prime}$ & $3159^{\prime} 43^{\prime \prime}$ \\
\hline 51 & 3017 & $4424^{\prime} 2^{\prime \prime}$ & $3159^{\prime} 26^{\prime \prime}$ & 106 & 8031 & $4421^{\prime} 12^{\prime \prime}$ & $3158^{\prime} 51^{\prime \prime}$ & 161 & 8046 & $4418^{\prime} 37^{\prime \prime}$ & $3159^{\prime} 43^{\prime \prime}$ \\
\hline 52 & 4017 & $4424^{\prime} 18^{\prime \prime}$ & $3159^{\prime} 23^{\prime \prime}$ & 107 & 1032 & $4421^{\prime} 28^{\prime \prime}$ & $320^{\prime} 42^{\prime \prime}$ & 162 & 9046 & $4418^{\prime} 51^{\prime \prime}$ & $3159^{\prime} 48^{\prime \prime}$ \\
\hline 53 & 1018 & $4418^{\prime} 46^{\prime \prime}$ & $3159^{\prime} 40^{\prime \prime}$ & 108 & 2032 & $4421^{\prime} 28^{\prime \prime}$ & $320^{\prime} 42^{\prime \prime}$ & 163 & 10046 & $4418^{\prime} 54^{\prime \prime}$ & $3159^{\prime} 48^{\prime \prime}$ \\
\hline 54 & 2018 & $4418^{\prime} 46^{\prime \prime}$ & $3159^{\prime} 40^{\prime \prime}$ & 109 & 3032 & $4421^{\prime} 29^{\prime \prime}$ & $320^{\prime} 41^{\prime \prime}$ & 164 & 11046 & $4418^{\prime} 55^{\prime \prime}$ & $3159^{\prime} 44^{\prime \prime}$ \\
\hline 55 & 1019 & $4418^{\prime} 47^{\prime \prime}$ & $3159^{\prime} 46^{\prime \prime}$ & 110 & 4032 & $4421^{\prime} 29^{\prime \prime}$ & $320^{\prime} 42^{\prime \prime}$ & 165 & 12046 & $4418^{\prime} 54^{\prime \prime}$ & $3159^{\prime} 43^{\prime \prime}$ \\
\hline No. & $\begin{array}{l}\text { Borehole } \\
\text { codes }\end{array}$ & Long. & Latt. & No. & $\begin{array}{l}\text { Borehole } \\
\text { codes }\end{array}$ & Long. & Latt. & No. & $\begin{array}{l}\text { Borehole } \\
\text { codes }\end{array}$ & Long. & Latt. \\
\hline 166 & 13046 & $4418^{\prime} 52^{\prime \prime}$ & $3159^{\prime} 42^{\prime \prime}$ & 222 & 1064 & $4417^{\prime} 32^{\prime \prime}$ & $328^{\prime} 13^{\prime \prime}$ & 278 & 2091 & $4421^{\prime} 19^{\prime \prime}$ & $320^{\prime} 42^{\prime \prime}$ \\
\hline 167 & 1047 & $4421^{\prime} 28^{\prime \prime}$ & $320^{\prime} 42^{\prime \prime}$ & 223 & 2064 & $4413^{\prime} 11^{\prime \prime}$ & $328^{\prime} 17^{\prime \prime}$ & 279 & 3091 & $4421^{\prime} 20^{\prime \prime}$ & $320^{\prime} 42^{\prime \prime}$ \\
\hline 168 & 2047 & $4421^{\prime} 29^{\prime \prime}$ & $320^{\prime} 42^{\prime \prime}$ & 224 & 1065 & $4417^{\prime} 54^{\prime \prime}$ & $327^{\prime} 43^{\prime \prime}$ & 280 & 4091 & $4421^{\prime} 18^{\prime \prime}$ & $320^{\prime} 42^{\prime \prime}$ \\
\hline 169 & 3047 & $4421^{\prime} 28^{\prime \prime}$ & $320^{\prime} 41^{\prime \prime}$ & 225 & 1066 & $4418^{\prime} 57^{\prime \prime}$ & $3159^{\prime} 44^{\prime \prime}$ & 281 & 5091 & $4418^{\prime} 48^{\prime \prime}$ & $3159^{\prime} 42^{\prime \prime}$ \\
\hline 170 & 4047 & $4421^{\prime} 28^{\prime \prime}$ & $320^{\prime} 42^{\prime \prime}$ & 226 & 2066 & $4418^{\prime} 58^{\prime \prime}$ & $3159^{\prime} 44^{\prime \prime}$ & 282 & 6091 & $4418^{\prime} 36^{\prime \prime}$ & $3159^{\prime} 29^{\prime \prime}$ \\
\hline 171 & 5047 & $4421^{\prime} 29^{\prime \prime}$ & $320^{\prime} 41^{\prime \prime}$ & 227 & 1067 & $4420^{\prime} 59^{\prime \prime}$ & $322^{\prime} 44^{\prime \prime}$ & 283 & 7091 & $4418^{\prime} 40^{\prime \prime}$ & $3159^{\prime} 54^{\prime \prime}$ \\
\hline 172 & 6047 & $4421^{\prime} 29^{\prime \prime}$ & $320^{\prime} 42^{\prime \prime}$ & 228 & 2067 & $4421^{\prime} 0^{\prime \prime}$ & $322^{\prime} 43^{\prime \prime}$ & 284 & 8091 & $4418^{\prime} 39^{\prime \prime}$ & $3159^{\prime} 45^{\prime \prime}$ \\
\hline 173 & 1048 & $4419^{\prime} 42^{\prime \prime}$ & $320^{\prime} 28^{\prime \prime}$ & 229 & 1068 & $4421^{\prime} 31^{\prime \prime}$ & $321^{\prime} 16^{\prime \prime}$ & 285 & 9091 & $4418^{\prime} 50^{\prime \prime}$ & $3159^{\prime} 49^{\prime \prime}$ \\
\hline 174 & 2048 & $4419^{\prime} 42^{\prime \prime}$ & $320^{\prime} 28^{\prime \prime}$ & 230 & 2068 & $4421^{\prime} 33^{\prime \prime}$ & $321^{\prime} 17^{\prime \prime}$ & 286 & 1092 & $4419^{\prime} 52^{\prime \prime}$ & $3159^{\prime} 58^{\prime \prime}$ \\
\hline 175 & 1049 & $4420^{\prime} 37^{\prime \prime}$ & $320^{\prime} 28^{\prime \prime}$ & 231 & 1069 & $4419^{\prime} 5^{\prime \prime}$ & $3159^{\prime} 43^{\prime \prime}$ & 287 & 2092 & $4419^{\prime} 52^{\prime \prime}$ & $3159^{\prime} 58^{\prime \prime}$ \\
\hline 176 & 2049 & $4420^{\prime} 36^{\prime \prime}$ & $320^{\prime} 27^{\prime \prime}$ & 232 & 2069 & $4419^{\prime} 5^{\prime \prime}$ & $3159^{\prime} 43^{\prime \prime}$ & 288 & 3092 & $4419^{\prime} 52^{\prime \prime}$ & $3159^{\prime} 58^{\prime \prime}$ \\
\hline 177 & 3049 & $4420^{\prime} 35^{\prime \prime}$ & $320^{\prime} 29^{\prime \prime}$ & 233 & 1070 & $4419^{\prime} 10^{\prime \prime}$ & $3159^{\prime} 38^{\prime \prime}$ & 289 & 1093 & $4418^{\prime} 54^{\prime \prime}$ & $3159^{\prime} 54^{\prime \prime}$ \\
\hline 178 & 4049 & $4420^{\prime} 36^{\prime \prime}$ & $320^{\prime} 29^{\prime \prime}$ & 234 & 2070 & $4419^{\prime} 10^{\prime \prime}$ & $3159^{\prime} 39^{\prime \prime}$ & 290 & 2093 & $4418^{\prime} 54^{\prime \prime}$ & $3159^{\prime} 54^{\prime \prime}$ \\
\hline 179 & 5049 & $4420^{\prime} 36^{\prime \prime}$ & $320^{\prime} 28^{\prime \prime}$ & 235 & 1071 & $4419^{\prime} 2^{\prime \prime}$ & $3159^{\prime} 44^{\prime \prime}$ & 291 & 1094 & $4421^{\prime} 48^{\prime \prime}$ & $320^{\prime} 43^{\prime \prime}$ \\
\hline 180 & 1050 & $4420^{\prime} 47^{\prime \prime}$ & $320^{\prime} 26^{\prime \prime}$ & 236 & 2071 & $4419^{\prime} 3^{\prime \prime}$ & $3159^{\prime} 45^{\prime \prime}$ & 292 & 2094 & $4421^{\prime} 40^{\prime \prime}$ & $321^{\prime} 11^{\prime \prime}$ \\
\hline 181 & 2050 & $4420^{\prime} 47^{\prime \prime}$ & $320^{\prime} 26^{\prime \prime}$ & 237 & 1072 & $4419^{\prime} 9^{\prime \prime}$ & $3159^{\prime} 38^{\prime \prime}$ & 293 & 1095 & $4418^{\prime} 42^{\prime \prime}$ & $322^{\prime} 17^{\prime \prime}$ \\
\hline
\end{tabular}


continued

\begin{tabular}{|c|c|c|c|c|c|c|c|c|c|c|c|}
\hline No. & $\begin{array}{l}\text { Borehole } \\
\text { codes }\end{array}$ & Long. & Latt. & No. & $\begin{array}{l}\text { Borehole } \\
\text { codes }\end{array}$ & Long. & Latt. & No. & $\begin{array}{l}\text { Borehole } \\
\text { codes }\end{array}$ & Long. & Latt. \\
\hline 182 & 1051 & $4419^{\prime} 6^{\prime \prime}$ & $3159^{\prime} 45^{\prime \prime}$ & 238 & 2072 & $4419^{\prime} 10^{\prime \prime}$ & $3159^{\prime} 37^{\prime \prime}$ & 294 & 2095 & $4418^{\prime} 43^{\prime \prime}$ & $322^{\prime} 17^{\prime \prime}$ \\
\hline 183 & 2051 & $4419^{\prime} 5^{\prime \prime}$ & $3159^{\prime} 45^{\prime \prime}$ & 239 & 1073 & $4418^{\prime} 40^{\prime \prime}$ & $3159^{\prime} 41^{\prime \prime}$ & 295 & 1096 & $4418^{\prime} 57^{\prime \prime}$ & $3159^{\prime} 48^{\prime \prime}$ \\
\hline 184 & 1052 & $4419^{\prime} 1^{\prime \prime}$ & $3159^{\prime} 45^{\prime \prime}$ & 240 & 2073 & $4418^{\prime} 40^{\prime \prime}$ & $3159^{\prime} 40^{\prime \prime}$ & 296 & 2096 & $4418^{\prime} 58^{\prime \prime}$ & $3159^{\prime} 48^{\prime \prime}$ \\
\hline 185 & 2052 & $4419^{\prime} 1^{\prime \prime}$ & $3159^{\prime} 44^{\prime \prime}$ & 241 & 1074 & $4419^{\prime} 8^{\prime \prime}$ & $3159^{\prime} 39^{\prime \prime}$ & 297 & 3096 & $4418^{\prime} 57^{\prime \prime}$ & $3159^{\prime} 48^{\prime \prime}$ \\
\hline 186 & 1053 & $4419^{\prime} 14^{\prime \prime}$ & $3159^{\prime} 47^{\prime \prime}$ & 242 & 2074 & $4419^{\prime} 8^{\prime \prime}$ & $3159^{\prime} 40^{\prime \prime}$ & 298 & 4096 & $4418^{\prime} 57^{\prime \prime}$ & $3159^{\prime} 49^{\prime \prime}$ \\
\hline 187 & 2053 & $4419^{\prime} 14^{\prime \prime}$ & $3159^{\prime} 47^{\prime \prime}$ & 243 & 1075 & $4419^{\prime} 9^{\prime \prime}$ & $3159^{\prime} 39^{\prime \prime}$ & 299 & 1097 & $4418^{\prime} 40^{\prime \prime}$ & $3159^{\prime} 53^{\prime \prime}$ \\
\hline 188 & 1054 & $4419^{\prime} 0^{\prime \prime}$ & $3159^{\prime} 44^{\prime \prime}$ & 244 & 2075 & $4419^{\prime} 9^{\prime \prime}$ & $3159^{\prime} 39^{\prime \prime}$ & 300 & 2097 & $4418^{\prime} 42^{\prime \prime}$ & $3159^{\prime} 54^{\prime \prime}$ \\
\hline 189 & 2054 & $4419^{\prime} 0^{\prime \prime}$ & $3159^{\prime} 45^{\prime \prime}$ & 245 & 1076 & $4419^{\prime} 9^{\prime \prime}$ & $3159^{\prime} 40^{\prime \prime}$ & 301 & 3097 & $4418^{\prime} 41^{\prime \prime}$ & $3159^{\prime} 54^{\prime \prime}$ \\
\hline 190 & 1055 & $4418^{\prime} 4^{\prime \prime}$ & $3159^{\prime} 28^{\prime \prime}$ & 246 & 2076 & $4419^{\prime} 9^{\prime \prime}$ & $3159^{\prime} 40^{\prime \prime}$ & 302 & 1098 & $4422^{\prime} 18^{\prime \prime}$ & $3159^{\prime} 37^{\prime \prime}$ \\
\hline 191 & 2055 & $4422^{\prime} 40^{\prime \prime}$ & $3158^{\prime} 14^{\prime \prime}$ & 247 & 1077 & $4419^{\prime} 3^{\prime \prime}$ & $3159^{\prime} 46^{\prime \prime}$ & 303 & 2098 & $4422^{\prime} 18^{\prime \prime}$ & $3159^{\prime} 38^{\prime \prime}$ \\
\hline 192 & 3055 & $4422^{\prime} 30^{\prime \prime}$ & $3158^{\prime} 43^{\prime \prime}$ & 248 & 2077 & $4419^{\prime} 4^{\prime \prime}$ & $3159^{\prime} 46^{\prime \prime}$ & 304 & 3098 & $4422^{\prime} 17^{\prime \prime}$ & $3159^{\prime} 38^{\prime \prime}$ \\
\hline 193 & 4055 & $4418^{\prime} 56^{\prime \prime}$ & $3159^{\prime} 45^{\prime \prime}$ & 249 & 1078 & $4419^{\prime} 9^{\prime \prime}$ & $3159^{\prime} 41^{\prime \prime}$ & 305 & 1099 & $4421^{\prime} 28^{\prime \prime}$ & $320^{\prime} 41^{\prime \prime}$ \\
\hline 194 & 1056 & $4421^{\prime} 4^{\prime \prime}$ & $3159^{\prime} 41^{\prime \prime}$ & 250 & 2078 & $4419^{\prime} 9^{\prime \prime}$ & $3159^{\prime} 41^{\prime \prime}$ & 306 & 2099 & $4421^{\prime} 4^{\prime \prime}$ & $320^{\prime} 41^{\prime \prime}$ \\
\hline 195 & 2056 & $4421^{\prime} 5^{\prime \prime}$ & $3159^{\prime} 43^{\prime \prime}$ & 251 & 1079 & $4421^{\prime} 12^{\prime \prime}$ & $320^{\prime} 29^{\prime \prime}$ & 307 & 3099 & $4421^{\prime} 28^{\prime \prime}$ & $320^{\prime} 41^{\prime \prime}$ \\
\hline 196 & 1057 & $4420^{\prime} 21^{\prime \prime}$ & $323^{\prime} 52^{\prime \prime}$ & 252 & 2079 & $4421^{\prime} 11^{\prime \prime}$ & $320^{\prime} 29^{\prime \prime}$ & 308 & 4099 & $4421^{\prime} 35^{\prime \prime}$ & $320^{\prime} 42^{\prime \prime}$ \\
\hline 197 & 2057 & $4420^{\prime} 23^{\prime \prime}$ & $323^{\prime} 51^{\prime \prime}$ & 253 & 1080 & $4421^{\prime} 12^{\prime \prime}$ & $320^{\prime} 54^{\prime \prime}$ & 309 & 5099 & $4419^{\prime} 3^{\prime \prime}$ & $3159^{\prime} 42^{\prime \prime}$ \\
\hline 198 & 3057 & $4420^{\prime} 20^{\prime \prime}$ & $323^{\prime} 52^{\prime \prime}$ & 254 & 2080 & $4421^{\prime} 13^{\prime \prime}$ & $320^{\prime} 52^{\prime \prime}$ & 310 & 1100 & $4419^{\prime} 26^{\prime \prime}$ & $324^{\prime} 2^{\prime \prime}$ \\
\hline 199 & 4057 & $4420^{\prime} 22^{\prime \prime}$ & $323^{\prime} 52^{\prime \prime}$ & 255 & 1081 & $4419^{\prime} 0^{\prime \prime}$ & $3159^{\prime} 46^{\prime \prime}$ & 311 & 2100 & $4419^{\prime} 28^{\prime \prime}$ & $324^{\prime} 2^{\prime \prime}$ \\
\hline 200 & 1058 & $4420^{\prime} 40^{\prime \prime}$ & $320^{\prime} 28^{\prime \prime}$ & 256 & 2081 & $4418^{\prime} 59^{\prime \prime}$ & $3159^{\prime} 46^{\prime \prime}$ & 312 & 1101 & $4418^{\prime} 30^{\prime \prime}$ & $322^{\prime} 30^{\prime \prime}$ \\
\hline 201 & 2058 & $4420^{\prime} 41^{\prime \prime}$ & $320^{\prime} 28^{\prime \prime}$ & 257 & 1082 & $4419^{\prime} 5^{\prime \prime}$ & $3159^{\prime} 46^{\prime \prime}$ & 313 & 2101 & $4418^{\prime} 30^{\prime \prime}$ & $322^{\prime} 31^{\prime \prime}$ \\
\hline 202 & 3058 & $4420^{\prime} 43^{\prime \prime}$ & $320^{\prime} 29^{\prime \prime}$ & 258 & 2082 & $4419^{\prime} 5^{\prime \prime}$ & $3159^{\prime} 46^{\prime \prime}$ & 314 & 3101 & $4418^{\prime} 31^{\prime \prime}$ & $322^{\prime} 32^{\prime \prime}$ \\
\hline 203 & 4058 & $4420^{\prime} 44^{\prime \prime}$ & $320^{\prime} 29^{\prime \prime}$ & 259 & 1083 & $4421^{\prime} 13^{\prime \prime}$ & $320^{\prime} 35^{\prime \prime}$ & 315 & 1102 & $4418^{\prime} 33^{\prime \prime}$ & $3159^{\prime} 53^{\prime \prime}$ \\
\hline 204 & 5058 & $4420^{\prime} 42^{\prime \prime}$ & $320^{\prime} 27^{\prime \prime}$ & 260 & 2083 & $4421^{\prime} 12^{\prime \prime}$ & $320^{\prime} 36^{\prime \prime}$ & 316 & 2102 & $4418^{\prime} 35^{\prime \prime}$ & $3159^{\prime} 51^{\prime \prime}$ \\
\hline 205 & 6058 & $4420^{\prime} 43^{\prime \prime}$ & $320^{\prime} 28^{\prime \prime}$ & 261 & 3083 & $4421^{\prime} 12^{\prime \prime}$ & $320^{\prime} 36^{\prime \prime}$ & 317 & 1103 & $4416^{\prime} 59^{\prime \prime}$ & $321^{\prime} 0^{\prime \prime}$ \\
\hline 206 & 7058 & $4420^{\prime} 41^{\prime \prime}$ & $320^{\prime} 26^{\prime \prime}$ & 262 & 4083 & $4421^{\prime} 12^{\prime \prime}$ & $320^{\prime} 36^{\prime \prime}$ & 318 & 2103 & $4416^{\prime} 54^{\prime \prime}$ & $320^{\prime} 57^{\prime \prime}$ \\
\hline 207 & 8058 & $4420^{\prime} 43^{\prime \prime}$ & $320^{\prime} 27^{\prime \prime}$ & 263 & 1084 & $4418^{\prime} 58^{\prime \prime}$ & $3159^{\prime} 46^{\prime \prime}$ & 319 & 3103 & $4416^{\prime} 52^{\prime \prime}$ & $321^{\prime} 4^{\prime \prime}$ \\
\hline 208 & 9058 & $4420^{\prime} 45^{\prime \prime}$ & $320^{\prime} 28^{\prime \prime}$ & 264 & 2084 & $4418^{\prime} 57^{\prime \prime}$ & $3159^{\prime} 46^{\prime \prime}$ & 320 & 1104 & $4423^{\prime} 33^{\prime \prime}$ & $320^{\prime} 13^{\prime \prime}$ \\
\hline 209 & 10058 & $4420^{\prime} 42^{\prime \prime}$ & $320^{\prime} 26^{\prime \prime}$ & 265 & 1085 & $4419^{\prime} 1^{\prime \prime}$ & $3159^{\prime} 46^{\prime \prime}$ & 321 & 2104 & $4423^{\prime} 32^{\prime \prime}$ & $320^{\prime} 14^{\prime \prime}$ \\
\hline 210 & 12058 & $4420^{\prime} 46^{\prime \prime}$ & $320^{\prime} 28^{\prime \prime}$ & 266 & 2085 & $4419^{\prime} 0^{\prime \prime}$ & $3159^{\prime} 46^{\prime \prime}$ & 322 & 3104 & $4423^{\prime} 32^{\prime \prime}$ & $320^{\prime} 13^{\prime \prime}$ \\
\hline 211 & 14058 & $4420^{\prime} 43^{\prime \prime}$ & $320^{\prime} 25^{\prime \prime}$ & 267 & 1086 & $4418^{\prime} 38^{\prime \prime}$ & $3152^{\prime} 54^{\prime \prime}$ & 323 & 1105 & $4420^{\prime} 35^{\prime \prime}$ & $321^{\prime} 37^{\prime \prime}$ \\
\hline 212 & 1059 & $4418^{\prime} 36^{\prime \prime}$ & $3159^{\prime} 24^{\prime \prime}$ & 268 & 2086 & $4418^{\prime} 38^{\prime \prime}$ & $3121^{\prime} 53^{\prime \prime}$ & 324 & 2105 & $4420^{\prime} 37^{\prime \prime}$ & $321^{\prime} 37^{\prime \prime}$ \\
\hline 213 & 2059 & $4418^{\prime} 36^{\prime \prime}$ & $3159^{\prime} 28^{\prime \prime}$ & 269 & 1087 & $4419^{\prime} 0^{\prime \prime}$ & $3159^{\prime} 46^{\prime \prime}$ & 325 & 1106 & $4420^{\prime} 42^{\prime \prime}$ & $3159^{\prime} 26^{\prime \prime}$ \\
\hline 214 & 1060 & $4420^{\prime} 12^{\prime \prime}$ & $322^{\prime} 43^{\prime \prime}$ & 270 & 2087 & $4418^{\prime} 59^{\prime \prime}$ & $3159^{\prime} 46^{\prime \prime}$ & 326 & 2106 & $4420^{\prime} 42^{\prime \prime}$ & $3159^{\prime} 26^{\prime \prime}$ \\
\hline 215 & 2060 & $4420^{\prime} 8^{\prime \prime}$ & $322^{\prime} 43^{\prime \prime}$ & 271 & 1088 & $4418^{\prime} 38^{\prime \prime}$ & $3159^{\prime} 40^{\prime \prime}$ & 327 & 1107 & $4421^{\prime} 9^{\prime \prime}$ & $320^{\prime} 29^{\prime \prime}$ \\
\hline 216 & 1061 & $4418^{\prime} 59^{\prime \prime}$ & $3159^{\prime} 44^{\prime \prime}$ & 272 & 2088 & $4418^{\prime} 38^{\prime \prime}$ & $3159^{\prime} 40^{\prime \prime}$ & 328 & 2107 & $4421^{\prime} 9^{\prime \prime}$ & $320^{\prime} 28^{\prime \prime}$ \\
\hline 217 & 2061 & $4418^{\prime} 58^{\prime \prime}$ & $3159^{\prime} 44^{\prime \prime}$ & 273 & 1089 & $4418^{\prime} 40^{\prime \prime}$ & $3159^{\prime} 40^{\prime \prime}$ & 329 & 1108 & $4418^{\prime} 46^{\prime \prime}$ & $322^{\prime} 34^{\prime \prime}$ \\
\hline 218 & 1062 & $4421^{\prime} 11^{\prime \prime}$ & $321^{\prime} 11^{\prime \prime}$ & 274 & 2089 & $4418^{\prime} 43^{\prime \prime}$ & $3159^{\prime} 40^{\prime \prime}$ & 330 & 2108 & $4418^{\prime} 47^{\prime \prime}$ & $322^{\prime} 20^{\prime \prime}$ \\
\hline 219 & 2062 & $4421^{\prime} 14^{\prime \prime}$ & $321^{\prime} 7^{\prime \prime}$ & 275 & 1090 & $4421^{\prime} 48^{\prime \prime}$ & $322^{\prime} 15^{\prime \prime}$ & 331 & 1109 & $4420^{\prime} 32^{\prime \prime}$ & $3159^{\prime} 4^{\prime \prime}$ \\
\hline 220 & 1063 & $4419^{\prime} 4^{\prime \prime}$ & $3159^{\prime} 45^{\prime \prime}$ & 276 & 2090 & $4421^{\prime} 48^{\prime \prime}$ & $322^{\prime} 14^{\prime \prime}$ & 332 & 2109 & $4420^{\prime} 29^{\prime \prime}$ & $3159^{\prime} 0^{\prime \prime}$ \\
\hline 221 & 2063 & $4419^{\prime} 3^{\prime \prime}$ & $3159^{\prime} 44^{\prime \prime}$ & 277 & 1091 & $4421^{\prime} 20^{\prime \prime}$ & $320^{\prime} 40^{\prime \prime}$ & 333 & 1110 & $4423^{\prime} 38^{\prime \prime}$ & $321^{\prime} 33^{\prime \prime}$ \\
\hline
\end{tabular}


continued

\begin{tabular}{|c|c|c|c|c|c|c|c|c|c|c|c|}
\hline No. & $\begin{array}{l}\text { Borehole } \\
\text { codes }\end{array}$ & Long. & Latt. & No. & $\begin{array}{l}\text { Borehole } \\
\text { codes }\end{array}$ & Long. & Latt. & No. & $\begin{array}{l}\text { Borehole } \\
\text { codes }\end{array}$ & Long. & Latt. \\
\hline 334 & 2110 & $4423^{\prime} 24^{\prime \prime}$ & $321^{\prime} 33^{\prime \prime}$ & 393 & 1137 & $4418^{\prime} 47^{\prime \prime}$ & $323^{\prime} 12^{\prime \prime}$ & 452 & 2160 & $4422^{\prime} 2^{\prime \prime}$ & $320^{\prime} 37^{\prime \prime}$ \\
\hline 335 & 1111 & $4420^{\prime} 11^{\prime \prime}$ & $3159^{\prime} 21^{\prime \prime}$ & 394 & 2137 & $4418^{\prime} 47^{\prime \prime}$ & $323^{\prime} 11^{\prime \prime}$ & 453 & 1161 & $4420^{\prime} 14^{\prime \prime}$ & $3245^{\prime} 28^{\prime \prime}$ \\
\hline 336 & 2111 & $4420^{\prime} 10^{\prime \prime}$ & $3159^{\prime} 21^{\prime \prime}$ & 395 & 1138 & $4421^{\prime} 12^{\prime \prime}$ & $321^{\prime} 55^{\prime \prime}$ & 454 & 2161 & $4420^{\prime} 17^{\prime \prime}$ & $3233^{\prime} 1^{\prime \prime}$ \\
\hline 337 & 1112 & $4421^{\prime} 16^{\prime \prime}$ & $321^{\prime} 11^{\prime \prime}$ & 396 & 2138 & $4421^{\prime} 12^{\prime \prime}$ & $321^{\prime} 54^{\prime \prime}$ & 455 & 3161 & $4420^{\prime} 19^{\prime \prime}$ & $3236^{\prime} 58^{\prime \prime}$ \\
\hline 338 & 2112 & $4421^{\prime} 16^{\prime \prime}$ & $321^{\prime} 4^{\prime \prime}$ & 397 & 1139 & $4420^{\prime} 57^{\prime \prime}$ & $321^{\prime} 7^{\prime \prime}$ & 456 & 4161 & $4420^{\prime} 16^{\prime \prime}$ & $3223^{\prime} 14^{\prime \prime}$ \\
\hline 339 & 1113 & $4422^{\prime} 55^{\prime \prime}$ & $3158^{\prime} 35^{\prime \prime}$ & 398 & 2139 & $4420^{\prime} 56^{\prime \prime}$ & $321^{\prime} 8^{\prime \prime}$ & 457 & 1162 & $4422^{\prime} 17^{\prime \prime}$ & $320^{\prime} 3^{\prime \prime}$ \\
\hline 340 & 2113 & $4422^{\prime} 54^{\prime \prime}$ & $3158^{\prime} 36^{\prime \prime}$ & 399 & 1140 & $4418^{\prime} 36^{\prime \prime}$ & $3159^{\prime} 50^{\prime \prime}$ & 458 & 2162 & $4422^{\prime} 16^{\prime \prime}$ & $320^{\prime} 4^{\prime \prime}$ \\
\hline 341 & 3113 & $4422^{\prime} 53^{\prime \prime}$ & $3158^{\prime} 36^{\prime \prime}$ & 400 & 2140 & $4418^{\prime} 36^{\prime \prime}$ & $3159^{\prime} 49^{\prime \prime}$ & 459 & 3162 & $4422^{\prime} 17^{\prime \prime}$ & $320^{\prime} 4^{\prime \prime}$ \\
\hline 342 & 1114 & $4422^{\prime} 7^{\prime \prime}$ & $323^{\prime} 34^{\prime \prime}$ & 401 & 1141 & $4418^{\prime} 35^{\prime \prime}$ & $3159^{\prime} 49^{\prime \prime}$ & 460 & 4162 & $4422^{\prime} 17^{\prime \prime}$ & $320^{\prime} 4^{\prime \prime}$ \\
\hline 343 & 1115 & $4418^{\prime} 33^{\prime \prime}$ & $3159^{\prime} 40^{\prime \prime}$ & 402 & 2141 & $4418^{\prime} 35^{\prime \prime}$ & $3159^{\prime} 49^{\prime \prime}$ & 461 & 1163 & $4420^{\prime} 3^{\prime \prime}$ & $322^{\prime} 32^{\prime \prime}$ \\
\hline 344 & 1116 & $4422^{\prime} 32^{\prime \prime}$ & $3158^{\prime} 31^{\prime \prime}$ & 403 & 1142 & $4418^{\prime} 47^{\prime \prime}$ & $323^{\prime} 12^{\prime \prime}$ & 462 & 2163 & $4420^{\prime} 3^{\prime \prime}$ & $322^{\prime} 32^{\prime \prime}$ \\
\hline 345 & 2116 & $4422^{\prime} 31^{\prime \prime}$ & $3158^{\prime} 29^{\prime \prime}$ & 404 & 2142 & $4418^{\prime} 47^{\prime \prime}$ & $323^{\prime} 11^{\prime \prime}$ & 463 & 1164 & $4421^{\prime} 29^{\prime \prime}$ & $320^{\prime} 42^{\prime \prime}$ \\
\hline 346 & 1117 & $4419^{\prime} 40^{\prime \prime}$ & $321^{\prime} 58^{\prime \prime}$ & 405 & 1143 & $4420^{\prime} 53^{\prime \prime}$ & $322^{\prime} 14^{\prime \prime}$ & 464 & 2164 & $4421^{\prime} 28^{\prime \prime}$ & $320^{\prime} 41^{\prime \prime}$ \\
\hline 347 & 2117 & $4419^{\prime} 40^{\prime \prime}$ & $321^{\prime} 58^{\prime \prime}$ & 406 & 2143 & $4420^{\prime} 52^{\prime \prime}$ & $322^{\prime} 14^{\prime \prime}$ & & & & \\
\hline 348 & 3117 & $4419^{\prime} 40^{\prime \prime}$ & $321^{\prime} 58^{\prime \prime}$ & 407 & 1144 & $4419^{\prime} 2^{\prime \prime}$ & $3159^{\prime} 52^{\prime \prime}$ & & & & \\
\hline 349 & 4117 & $4419^{\prime} 40^{\prime \prime}$ & $321^{\prime} 58^{\prime \prime}$ & 408 & 2144 & $4419^{\prime} 2^{\prime \prime}$ & $3159^{\prime} 51^{\prime \prime}$ & & & & \\
\hline 350 & 5117 & $4419^{\prime} 40^{\prime \prime}$ & $321^{\prime} 58^{\prime \prime}$ & 409 & 1145 & $4418^{\prime} 51^{\prime \prime}$ & $323^{\prime} 13^{\prime \prime}$ & & & & \\
\hline 351 & 6117 & $4419^{\prime} 40^{\prime \prime}$ & $321^{\prime} 58^{\prime \prime}$ & 410 & 2145 & $4418^{\prime} 51^{\prime \prime}$ & $323^{\prime} 12^{\prime \prime}$ & & & & \\
\hline 352 & 7117 & $4419^{\prime} 40^{\prime \prime}$ & $321^{\prime} 58^{\prime \prime}$ & 411 & 1146 & $4418^{\prime} 46^{\prime \prime}$ & $3159^{\prime} 49^{\prime \prime}$ & & & & \\
\hline 353 & 8117 & $4419^{\prime} 40^{\prime \prime}$ & $321^{\prime} 58^{\prime \prime}$ & 412 & 2146 & $4418^{\prime} 46^{\prime \prime}$ & $3159^{\prime} 50^{\prime \prime}$ & & & & \\
\hline 354 & 1118 & $4420^{\prime} 14^{\prime \prime}$ & $322^{\prime} 51^{\prime \prime}$ & 413 & 1147 & $4419^{\prime} 56^{\prime \prime}$ & $321^{\prime} 49^{\prime \prime}$ & & & & \\
\hline 355 & 2118 & $4420^{\prime} 14^{\prime \prime}$ & $322^{\prime} 50^{\prime \prime}$ & 414 & 2147 & $4419^{\prime} 56^{\prime \prime}$ & $321^{\prime} 51^{\prime \prime}$ & & & & \\
\hline 356 & 1119 & $4420^{\prime} 57^{\prime \prime}$ & $320^{\prime} 2^{\prime \prime}$ & 415 & 1148 & $4418^{\prime} 59^{\prime \prime}$ & $323^{\prime} 58^{\prime \prime}$ & & & & \\
\hline 357 & 2119 & $4420^{\prime} 58^{\prime \prime}$ & $320^{\prime} 1^{\prime \prime}$ & 416 & 2148 & $4418^{\prime} 58^{\prime \prime}$ & $323^{\prime} 40^{\prime \prime}$ & & & & \\
\hline 358 & 1120 & $4422^{\prime} 13^{\prime \prime}$ & $320^{\prime} 1^{\prime \prime}$ & 417 & 3148 & $4419^{\prime} 0^{\prime \prime}$ & $323^{\prime} 46^{\prime \prime}$ & & & & \\
\hline 359 & 2120 & $4422^{\prime} 14^{\prime \prime}$ & $320^{\prime} 1^{\prime \prime}$ & 418 & 1149 & $4419^{\prime} 36^{\prime \prime}$ & $325^{\prime} 14^{\prime \prime}$ & & & & \\
\hline 360 & 1121 & $4420^{\prime} 21^{\prime \prime}$ & $320^{\prime} 19^{\prime \prime}$ & 419 & 2149 & $4419^{\prime} 42^{\prime \prime}$ & $325^{\prime} 13^{\prime \prime}$ & & & & \\
\hline 361 & 2121 & $4420^{\prime} 22^{\prime \prime}$ & $320^{\prime} 19^{\prime \prime}$ & 420 & 3149 & $4419^{\prime} 39^{\prime \prime}$ & $325^{\prime} 14^{\prime \prime}$ & & & & \\
\hline 362 & 1122 & $4418^{\prime} 59^{\prime \prime}$ & $3159^{\prime} 43^{\prime \prime}$ & 421 & 1150 & $4419^{\prime} 36^{\prime \prime}$ & $323^{\prime} 19^{\prime \prime}$ & & & & \\
\hline 363 & 2122 & $4418^{\prime} 57^{\prime \prime}$ & $3159^{\prime} 28^{\prime \prime}$ & 422 & 2150 & $4419^{\prime} 37^{\prime \prime}$ & $323^{\prime} 18^{\prime \prime}$ & & & & \\
\hline 364 & 1123 & $4418^{\prime} 44^{\prime \prime}$ & $326^{\prime} 4^{\prime \prime}$ & 423 & 3150 & $4419^{\prime} 35^{\prime \prime}$ & $323^{\prime} 18^{\prime \prime}$ & & & & \\
\hline 365 & 2123 & $4418^{\prime} 36^{\prime \prime}$ & $327^{\prime} 14^{\prime \prime}$ & 424 & 1151 & $4421^{\prime} 44^{\prime \prime}$ & $322^{\prime} 24^{\prime \prime}$ & & & & \\
\hline 366 & 1124 & $4418^{\prime} 6^{\prime \prime}$ & $3211^{\prime} 28^{\prime \prime}$ & 425 & 2151 & $4421^{\prime} 42^{\prime \prime}$ & $322^{\prime} 23^{\prime \prime}$ & & & & \\
\hline 367 & 2124 & $4419^{\prime} 50^{\prime \prime}$ & $320^{\prime} 5^{\prime \prime}$ & 426 & 3151 & $4421^{\prime} 45^{\prime \prime}$ & $322^{\prime} 22^{\prime \prime}$ & & & & \\
\hline 368 & 1125 & $4419^{\prime} 31^{\prime \prime}$ & $320^{\prime} 53^{\prime \prime}$ & 427 & 4151 & $4421^{\prime} 47^{\prime \prime}$ & $322^{\prime} 23^{\prime \prime}$ & & & & \\
\hline 369 & 2125 & $4419^{\prime} 32^{\prime \prime}$ & $320^{\prime} 56^{\prime \prime}$ & 428 & 5151 & $4421^{\prime} 45^{\prime \prime}$ & $322^{\prime} 26^{\prime \prime}$ & & & & \\
\hline 370 & 1126 & $4420^{\prime} 20^{\prime \prime}$ & $3159^{\prime} 51^{\prime \prime}$ & 429 & 6151 & $4421^{\prime} 43^{\prime \prime}$ & $322^{\prime} 26^{\prime \prime}$ & & & & \\
\hline 371 & 2126 & $4420^{\prime} 21^{\prime \prime}$ & $3159^{\prime} 51^{\prime \prime}$ & 430 & 1152 & $4421^{\prime} 8^{\prime \prime}$ & $322^{\prime} 45^{\prime \prime}$ & & & & \\
\hline 372 & 1127 & $4420^{\prime} 32^{\prime \prime}$ & $321^{\prime} 34^{\prime \prime}$ & 431 & 2152 & $4421^{\prime} 0^{\prime \prime}$ & $321^{\prime} 15^{\prime \prime}$ & & & & \\
\hline 373 & 2127 & $4420^{\prime} 32^{\prime \prime}$ & $321^{\prime} 33^{\prime \prime}$ & 432 & 3152 & $4421^{\prime} 28^{\prime \prime}$ & $321^{\prime} 16^{\prime \prime}$ & & & & \\
\hline 374 & 1128 & $4418^{\prime} 57^{\prime \prime}$ & $3159^{\prime} 30^{\prime \prime}$ & 433 & 4152 & $4419^{\prime} 5^{\prime \prime}$ & $3159^{\prime} 44^{\prime \prime}$ & & & & \\
\hline 375 & 1129 & $4419^{\prime} 16^{\prime \prime}$ & $3159^{\prime} 19^{\prime \prime}$ & 434 & 5152 & $4419^{\prime} 4^{\prime \prime}$ & $3159^{\prime} 42^{\prime \prime}$ & & & & \\
\hline 376 & 2129 & $4419^{\prime} 17^{\prime \prime}$ & $3159^{\prime} 18^{\prime \prime}$ & 435 & 1153 & $4419^{\prime} 8^{\prime \prime}$ & $3159^{\prime} 38^{\prime \prime}$ & & & & \\
\hline 377 & 1130 & $4419^{\prime} 18^{\prime \prime}$ & $323^{\prime} 56^{\prime \prime}$ & 436 & 2153 & $4419^{\prime} 11^{\prime \prime}$ & $3158^{\prime} 52^{\prime \prime}$ & & & & \\
\hline
\end{tabular}


continued

\begin{tabular}{|c|c|c|c|c|c|c|c|c|c|c|c|}
\hline No. & $\begin{array}{l}\text { Borehole } \\
\text { codes }\end{array}$ & Long. & Latt. & No. & $\begin{array}{l}\text { Borehole } \\
\text { codes }\end{array}$ & Long. & Latt. & No. & $\begin{array}{l}\text { Borehole } \\
\text { codes }\end{array}$ & Long. & Latt. \\
\hline 378 & 2130 & $4419^{\prime} 18^{\prime \prime}$ & $323^{\prime} 55^{\prime \prime}$ & 437 & 1154 & $4423^{\prime} 37^{\prime \prime}$ & $321^{\prime} 36^{\prime \prime}$ & & & & \\
\hline 379 & 1131 & $4419^{\prime} 51^{\prime \prime}$ & $3159^{\prime} 6^{\prime \prime}$ & 438 & 2154 & $4423^{\prime} 36^{\prime \prime}$ & $321^{\prime} 37^{\prime \prime}$ & & & & \\
\hline 380 & 2131 & $4419^{\prime} 50^{\prime \prime}$ & $3159^{\prime} 5^{\prime \prime}$ & 439 & 1155 & $4422^{\prime} 48^{\prime \prime}$ & $3158^{\prime} 35^{\prime \prime}$ & & & & \\
\hline 381 & 1132 & $4421^{\prime} 41^{\prime \prime}$ & $320^{\prime} 41^{\prime \prime}$ & 440 & 2155 & $4422^{\prime} 48^{\prime \prime}$ & $3158^{\prime} 38^{\prime \prime}$ & & & & \\
\hline 382 & 2132 & $4421^{\prime} 40^{\prime \prime}$ & $320^{\prime} 40^{\prime \prime}$ & 441 & 3155 & $4422^{\prime} 51^{\prime \prime}$ & $3158^{\prime} 39^{\prime \prime}$ & & & & \\
\hline 383 & 1133 & $4419^{\prime} 11^{\prime \prime}$ & $3159^{\prime} 56^{\prime \prime}$ & 442 & 4155 & $4422^{\prime} 52^{\prime \prime}$ & $3158^{\prime} 37^{\prime \prime}$ & & & & \\
\hline 384 & 2133 & $4417^{\prime} 28^{\prime \prime}$ & $320^{\prime} 35^{\prime \prime}$ & 443 & 1156 & $4418^{\prime} 50^{\prime \prime}$ & $322^{\prime} 24^{\prime \prime}$ & & & & \\
\hline 385 & 3133 & $4417^{\prime} 29^{\prime \prime}$ & $320^{\prime} 31^{\prime \prime}$ & 444 & 2156 & $4418^{\prime} 49^{\prime \prime}$ & $322^{\prime} 23^{\prime \prime}$ & & & & \\
\hline 386 & 1134 & $4419^{\prime} 6^{\prime \prime}$ & $3159^{\prime} 50^{\prime \prime}$ & 445 & 1157 & $4418^{\prime} 43^{\prime \prime}$ & $321^{\prime} 30^{\prime \prime}$ & & & & \\
\hline 387 & 2134 & $4419^{\prime} 6^{\prime \prime}$ & $3159^{\prime} 50^{\prime \prime}$ & 446 & 2157 & $4418^{\prime} 43^{\prime \prime}$ & $321^{\prime} 32^{\prime \prime}$ & & & & \\
\hline 388 & 1135 & $444^{\prime} 34^{\prime \prime}$ & $316^{\prime} 11^{\prime \prime}$ & 447 & 1158 & $4420^{\prime} 0^{\prime \prime}$ & $320^{\prime} 5^{\prime \prime}$ & & & & \\
\hline 389 & 2135 & $444^{\prime} 10^{\prime \prime}$ & $316^{\prime} 47^{\prime \prime}$ & 448 & 2158 & $4419^{\prime} 59^{\prime \prime}$ & $320^{\prime} 5^{\prime \prime}$ & & & & \\
\hline 390 & 3135 & $443^{\prime} 7^{\prime \prime}$ & $3111^{\prime} 20^{\prime \prime}$ & 449 & 1159 & $4419^{\prime} 57^{\prime \prime}$ & $3158^{\prime} 56^{\prime \prime}$ & & & & \\
\hline 391 & 1136 & $4418^{\prime} 42^{\prime \prime}$ & $3159^{\prime} 51^{\prime \prime}$ & 450 & 2159 & $4419^{\prime} 57^{\prime \prime}$ & $3158^{\prime} 56^{\prime \prime}$ & & & & \\
\hline 392 & 2136 & $4418^{\prime} 42^{\prime \prime}$ & $3159^{\prime} 51^{\prime \prime}$ & 451 & 1160 & $4422^{\prime} 3^{\prime \prime}$ & $320^{\prime} 36^{\prime \prime}$ & & & & \\
\hline
\end{tabular}

\section{References}

Al-Amoudi OS, Alawi K, Maslehuddin M (2015) Performance of corrosion inhibitors under the combined effect of chloride and sulfate contamination in concrete. In: Corrosion 2015, vol 1, Dallas, TX

Ali TS (2017) Effect of ground water sodium chloride attack on reinforced concrete footings. J Geotech Eng 4(1):1-7

Ali TS, Fakhraldin M K(2016) Soil parameters analysis of AlNajaf City in Iraq: case study. J Geotech Eng 3(1). ISSN: 2394-1987 (online)

Allen C (2005) Physical and chemical characteristics of soils foaming on boulder tops Karkevagge, Sweden. J Am Soil Sci Soc 69:148-158

Al-Maliki LAJ, Al-Mamoori SK, El-Tawil K, Hussain HM, AlAnsari N, Al Ali MJ (2018) Distribution of bearing capacity for An-Najaf and Kufa cities using GIS. Engineering 10:262-269

Al-Mamoori SK (2017) Gypsum content horizontal and vertical distribution of An-Najaf and Al-Kufa cities' soil by using GIS. Basrah J Eng Sci 17(1):48-60

Al-Mamoori SK, Al-Maliki LA, Hussain HM, Al-Ali MJ (2017) Distribution of sulfate and organic matter in An-Najaf and Al-Kufa cities' soil using GIS. Kufa J Eng 9(3):92-111

Atyah A, Muir WJ (2013) Study of some physical, chemical and engineering properties of selected soil for the site of university buildings at the University of Babylon. J Babylon Univ Eng Sci 21(2)

Bonic Z, Toplicic-Curcic GA, Davidović N, Jelena S (2015) Damage of concrete and reinforcement of reinforced-concrete foundations caused by environmental effects. Proc Eng 117:411-418

Emmanuel AO, Oladipo FA, Ogunsanmi OE (2012) Investigation of salinity effect on compressive strength of reinforced concrete. J Sustain Dev. https://doi.org/10.5539/jsd. v5n6p74

Glasser FP, Marchand J, Samson E (2008) Durability of concrete degradation phenomena involving detrimental chemical reactions. Cem Concr Res 38(2):226-246

Hirao H, Yamada K, Takahashi H, Zibara H (2005) Chloride binding of cement estimated by binding isotherms of hydrates. J Adv Concr Technol 3(1):77-84

Karthick SP, Muralidharan S, Saraswathy V, Kwon S-J (2016) Effect of different alkali salt additions on concrete durability property. J Struct Integr Maint 1(1):35-42

Mahmoud RA, Jaafar EMA (2017) Study of sulfur, chloride and carbonate ratios and their effect on geometric behavior of shallow loading layers in selected areas of Basra City/ Southern Iraq. Univ Babylon J Pure Appl Sci 1(25):160-171

Mallick DV, Tawil MM, Shibani AA (2018) Durability of foundation concrete in an aggressive environment. IABSE reports. Persistenter link: https://doi.org/10.5169/seals44219

Maslehuddin M, Al-Zahrani MM, Ibrahim M, Al-Mehthel MH, Al-Idi SH (2007) Effect of chloride concentration in soil on reinforcement corrosion. Constr Build Mater 21:1825-1832

National Center for Construction Laboratories \& Research (NCCLR) (2016) Investigation reports

Niville N (1995) Chloride attack of reinforced concrete: an overview. Mater Struct 28:63-70

Oh BH, Jang SY (2005) Experimental investigation on the threshold chloride concentration for corrosion initiation in reinforced concrete structures. In: 18th international conference on structural mechanics in reactor technology (SMiRT 18), SMiRT18-H11-1, Beijing, China, 7-12 Aug 2005

Shahrour I, Alileche L, Alfurjani A (2007) Smart cities: system and tools used for the digital modelling of physical urban systems. In: 2017 sensors networks smart and emerging technologies (SENSET). IEEE 